\title{
Analytic Evaluation of Contention Protocols Used in Distributed Real-Time Systems*
}

\author{
KANG SHIN \\ Real-Time Computing Laboratory \\ Department of Electrical Engineering and Computer Science \\ The University of Michigan \\ Ann Arbor, $M I$ 48105-2122 \\ CHAO-JU HOU \\ Department of Electrical and Computer Engineering \\ The University of Wisconsin - Madison \\ Madison, WI 53706-1691
}

kgshin@eecs.umich,edu

Received May 1, 1991

Editor:

\begin{abstract}
The probability of a station failing to deliver packets before their deadlines, called the probability of dynamic failure, $P_{d y n}$, is an important measure for the communication subsystem of a distributed realtime system. Another closely-related performance measure is the $\varepsilon$-bounded delivery time, $T_{\varepsilon}$, which is defined as the least time needed to deliver a packet with probability greater than $1-\varepsilon$. Using $P_{d y n}$ and $T_{\varepsilon}$, we comparatively evaluate four contention protocols often used in distributed real-time systems: (i) the token passing protocol and its priority-based variation (called the token scheduling protocol), and (ii) the $P_{i}$-persistent protocol and a priority-based variation thereof. The communication subsystem equipped with different contention protocols is modeled first as embedded Markov chains. Then, we derive the probability distributions of access delay, from which $P_{d y n}$ and $T_{\varepsilon}$ can be calculated. The blocking probability, $Q_{\dot{z}}$, can also be derived from the access delay distribution. These measures are derived first under the assumption of a single buffer at each station. The single-buffer model is then extended to the multiple-buffer case. The effects of buffer size on $P_{d y n}, T_{\varepsilon}$, and $Q_{i}$, and the performance improvement with multiple buffers are analyzed over a wide range of network traffic.
\end{abstract}

Keywords: Deadlines, real-time systems, contention protocols, access delay, embedded Markov chains, queneing theory, performance metrics, performance evaluation.

\section{Introduction}

Due to their potential for high performance and high reliability, distributed computing systems are being used for an increasing number of real-time applications which execute computational tasks. Real-time tasks often have to communicate with one another via a common communication medium in order to collectively perform some useful function. Each real-time task must be completed before its deadline, and thus, the messages generated/needed by this task must be delivered/received in a timely manner.

The work reported in this paper was supported in part by the ONR under Grant N00014-92-J-1080. Any opinions, findings, and conclusions or recommendations expressed in this paper are those of the authors and do not necessarily reflect the views of the ONR. 
Contention protocols are used to regulate the access of all stations - where real-time tasks are executed - to the common medium as well as to support efficient sharing of the medium. Any contention protocol used for real-time applications must therefore minimize the probability of a packet missing its delivery deadline by properly arbitrating access to the communication medium.

Contention protocols have been studied extensively for general-purpose distributed systems, such as the token-passing protocol [4], [3], [15], [24], [5], [8] and the CSMA/CD protocol [26], [21], [2], [1], [28], [14]. See [22] or [23] for a good survey. The protocols treated in these papers were originally developed for data transmission without regard to real-time services. Moreover, all but the results reported in [24], [8], [14] are concerned only with the evaluation of some average performance measures, such as aggregate throughput, average packet delay, and channel utilization. Although these average measures provide a useful yardstick for system performance, they are inadequate in assessing real-time performances, since the timing requirement of each individual task/message is not considered.

Recently, considerable attention has been paid to the development/modification of protocols to handle time-constrained communications. Manfield [13] proposed and analyzed a priority-polling system where high-priority messages are assumed to arrive at a single queue, and the queue holding high-priority messages and the other queues holding low-priority messages are alternately polled. Low-priority queues are polled cyclically between high-priority polls. In [6], he also presented and analyzed another prioritypolling scheme where the system is assumed to instantaneously detect the occurrence of a high-priority event. Rubin and Tsai [16] investigated a message-priority-based token scheme where different service disciplines are employed for different classes of messages. Tight upper and lower bounds on the mean message delays were obtained. In another paper [27], they presented and analyzed two related token schemes which provide priority services to high-priority messages, and restrict the channel access of low-priority messages. Although these papers aimed at the delivery of high-priority (time-constrained) packets with a prescribed access delay, their analyses focused on the mean delay experienced by messages of different priorities, rather than the delay experienced by each message.

Using the fraction of packets missing their deadlines as a performance measure, Zhao et al. developed a virtual time CSMA protocol [29] and a sliding window protocol [30], both of which are based on the minimum-laxity-first policy for scheduling timeconstrained packets. Strosnider et al. [20], [19] made use of the IEEE 802.5 Token Ring Standard to realize the token ring scheduling protocol which can support up to 8 different priority levels and provide highly responsive and deterministic services for timeconstrained messages. Kurose et. al [12] determined (based on a semi-Markov decision model) the various operational parameters for the time window protocol to optimize its time-constrained performance. However, the above work (perhaps except for [12]) relied solely on simulations to show the timing property of their proposed protocols. Little has been reported on the analytic evaluation of real-time contention protocols with respect to measures suitable for real-time applications, such as the probability of each packet missing its deadline. We will in this paper counter this problem by analytically 
evaluating the performance of four contention protocols for real-time applications on a per-packet basis.

One important performance measure for any real-time communication subsystem is the probability of a station failing to deliver packets before their deadlines, called the probability of dynamic failure, $P_{d y n}[11],[17],[18]$. Another related performance measure is the $\varepsilon$-bounded delivery time, $T_{\varepsilon}$, which is defined as the least time needed to deliver a packet (measured since its arrival) with probability greater than $1-\varepsilon$ [18]. These two measures are derived for both non priority-based and priority-based token-type protocols. The former is often referred to as the token passing protocol, and the latter as the token scheduling protocol [20], [19]. The $P_{i}$-persistent protocol and a priority variation thereof are also analyzed using $P_{d y n}$ and $T_{\mathcal{E}}$.

A communication subsystem with different contention protocols is modeled as embedded Markov chains. The probability distribution of the medium access delay $D_{A}$ - the time elapsed from the arrival to the beginning of transmission of a packet - is then derived. Both $P_{d y n}$ and $T_{\varepsilon}$ can be calculated from the distribution of access delay. The probability of packet rejection, $Q_{i}$, can also be derived from the access delay distribution. These measures are then used to evaluate the performance of four contention protocols. The derivation is first based on the assumption that each station has only a single buffer. This assumption greatly reduces modeling complexity, yet the results so derived can still represent the performance of contention protocols, with respect to the ability in regulating medium accesses according to the priority of the packet waiting at each station. Then, we extend the single-buffer model to the multiple-buffer case, and evaluate (i) the performance improvement over the single-buffer case, and (ii) the effects of buffer size on $P_{d y n}, T_{\varepsilon}$, and $Q_{i}$.

The remainder of this paper is organized as follows. The system model used and the contention protocols under consideration are described in Section 2. The relations among $P_{d y n}, T_{\varepsilon}$, and the distribution of access delay are also addressed there. In Section 3 , we develop performance models for the contention protocols under the single-buffer assumption. The probability distribution of access delay is then derived. Section 4 extends the single-buffer model to the multiple-buffer case. Section 5 presents numerical results of protocol evaluation in terms of $P_{d y n}, T_{\varepsilon}$, and $Q_{i}$. We also simulated the four protocols in order to validate analytic models. The paper concludes with Section 6.

\section{System Model, Performance Measures, and Protocols}

\subsection{System Model}

The system under consideration is homogeneous and consists of $N$ stations. Packet arrivals at station $i$ are assumed to follow a Poisson process with rate $\lambda_{i}$, and are independent of those of other stations. Packets are assumed to be of fixed length, each requiring $m$ units of time for transmission. There are $p$ priority levels for packets with 1 being the highest. Packets arriving at each station are classified into different priority levels according to their laxities. (Note that the laxity, $\ell$, of a packet is defined as the latest time a packet must start transmission in order to meet its deadline.) For example, 
a packet with laxity $\ell$ is assigned priority level $k$ when $\ell \in\left[\ell_{k-1}, \ell_{k}\right)$, where $\ell_{k-1}<\ell_{k}$, $1 \leq k \leq p, \ell_{0} \triangleq 0$, and the values of $\ell_{k}$ 's depends on the distribution of packet laxity and the application-dependent criterion. Under the assumption that packet arrivals are independent of one another, arrivals of priority $-k$ packets at station $i$ form a Poisson process with rate $\lambda_{i k}$, where $\frac{\lambda_{i k}}{\lambda_{i}}$ is the portion of priority-k packets arriving at station $i$.

Under the single-buffer assumption, each station can have at most one outstanding packet at any time, so those packets arriving at an already-occupied station will be rejected. One can then concentrate on the access delay experienced by a packet due to contention with other stations in accessing the common medium.

In the multiple-buffer model, there are $L$ buffers in each station. For simplicity, only two priority levels are assumed to exist. This is not a limiting assumption, since it can be easily extended to the case of more than two priority levels. Moreover, the results obtained for high-priority packets under the two priority-level assumption can also be used to represent the delay experienced by high-priority packets in case of $p$-priority levels $(p \geq 3$ ) by grouping low $p-1$ levels into one level.

For non priority-based protocols, packets are queued and served on a first-comefirst-served basis. For priority-based protocols, however, a buffer of size $L_{1}$ is used to store high-priority packets so that at most $L_{1}$ high-priority packets may be held at any time in each station. A high-priority packet arriving at a station whose $L_{1}$ buffers are all occupied is rejected. Another queue of size $L_{2}=L-L_{1}$ is set aside to hold low-priority packets. Since there must be an upper bound on the number of outstanding high-priority packets in the system in order to guarantee the delivery of every high-priority packet before its deadline, the value of $L_{1}$ is usually small. Low-priority packets at a station can compete with other stations for medium access only in the absence of high-priority packets waiting at this station. At most one packet can be transmitted during each possession of the token, since packets are prioritized according to their laxities, and thus should compete for medium access on a per-packet basis. (This is often termed as a limited service discipline).

\subsection{Performance Measures}

Instead of using the usual average measures, we will use $P_{d y n}$ and $T_{\varepsilon}$ to evaluate the performance of contention protocols. These measures can be readily calculated from the cumulative distribution of access delay. Specifically, given the distribution of access delay experienced by priority $-k$ packets, $P\left(D_{A}^{<k>} \leq t\right)$, one can calculate the conditional probability of dynamic failure given the laxity $\ell$ of a priority- $k$ packet as $P_{d y n \mid \ell}^{<k>} \equiv 1-P\left(D_{A}^{<k>} \leq \ell\right)$. The calculation of the probability of dynamic failure for priority- $k$ packets, $P_{d y n}^{<k>}$, depends on how the laxity of a packet is converted to its priority. For example, let $P_{\ell a x}(t), t \geq 0$, denote the probability density of packet laxity, then $P_{d y n_{n}}^{<k>}$ can be calculated as:

$$
P_{d y n}^{<k>}=\int_{\ell_{k-1}}^{\ell_{k}} P_{d y n \mid t}^{<k>} P_{\ell a x}(t) d t=\int_{\ell_{k-1}}^{\ell_{k}} P\left(D_{A}^{<k>}>t\right) P_{\ell a x}(t) d t .
$$


On the other hand, the $\varepsilon$-bounded delivery time, $T_{\varepsilon}^{<k>}$, for priority- $k$ packets is computed as the smallest $t$ such that $P_{d y n \mid t}^{<k>} \leq \varepsilon$. In other words, $T_{\varepsilon}^{<k>}$ is the upper bound of packet laxity if a priority $-k$ packet has to be delivered before its deadline with probability $1-\varepsilon$.

\subsection{Operations of the Four Contention Protocols}

In the token passing protocol, a token is passed around the stations, and any station which desires to use the medium has to capture the token. After using the medium, the station releases the token. The station in the downstream neighboring the current medium master gets the highest priority. If this station has packets to transmit, it seizes the token; otherwise, the token is passed on to the next (logically) adjacent station. Fair access to the bus is one merit of this protocol, but a packet with the highest priority (e.g., minimum laxity) may have to wait until all the packets in its upstream stations are transmitted, and thus, may get delayed and/or even miss its deadline.

The token scheduling protocol [20], [19] is designed to alleviate the above drawback by restricting fairness to packets only within the same priority level. When there are outstanding high-priority packets, low-priority packets should wait until the high-priority packets have been transmitted. A token with a reservation field is passed around the stations on the bus. Each station examines the reservation field of the token as it passes through the station and inserts the priority of its outstanding packet (if any) if and only if its priority is higher than the one currently in the reservation field. The priority recorded in the reservation field thus reflects the highest priority of those outstanding packets in the system. A station can capture the token and transmit its packets only after the token returns to the station with its registered priority. This guarantees the station with the highest-priority packet to gain medium access. We will develop analytic models to quantitatively evaluate these two protocols.

The $P_{i}$-persistent applies to slotted-time mediums and stations can start transmitting packets only at the beginning of each time slot. A station with an outstanding packet senses the medium. If the medium is idle, the station transmits the packet with a probability $p_{i}$. With a probability $1-p_{i}$ the station defers until the next slot. The process is repeated until the packet has been transmitted. If the station initially senses the medium busy, it waits until the next slot and applies the above procedure. One simple prioritybased variation of the $P_{i}$-persistent protocol is to assign distinct persistent probabilities to different priorities [25]; a station with a higher-priority packet persists to transmit this packet with a higher probability. Under light traffic, the average medium access delay of this protocol is known to be smaller than that of many other protocols. However, increasing packet collisions degrade system performance as traffic gets heavier. Moreover, a packet could get delayed indefinitely, because of the large standard deviation in delay over a wide range of traffic. We will examine the impact of the unbounded access delay of this protocol on time-critical applications. 


\section{Access Delay Distributions for the Single-Buffer Case}

As mentioned in Section 2, the key step in deriving both $P_{d y n}$ and $T_{\varepsilon}$ is to derive the distribution of access delay. In this section, we will derive this distribution using a single-buffer system model. We will in Section 4 extend this model to the multiplebuffer case.

\subsection{Token Passing Protocol}

In order to derive the distribution of access delay for the token passing protocol, we must first derive another quantity of interest, called the cycle time. The cycle time is defined as the time required for the token to cycle through all stations once, transmitting all packets, if any, in each station buffer at the time the station captures the token.

\subsubsection{Definition and Transition of States}

The first step of our derivation is to define an embedded Markov chain describing the system. Define the state as $\vec{n}=\left(n_{1}, n_{2}, \ldots, n_{N}\right)$ where $n_{i}(1 \leq i \leq N)$ is the station buffer occupancy seen by the token as it cycles through all stations, or

$$
n_{i}=\left\{\begin{array}{l}
0 \text { if the buffer is empty as the token comes to station } i \\
1 \text { if the buffer is full as the token comes to station } i .
\end{array}\right.
$$

The token is assumed to start cycling from a particular station. For a homogeneous system, it does not matter which station is chosen as the starting point. Moreover, we are interested in the state of the system not at some particular time instant, but as the token travels through the stations. Whenever the token comes to station $i$, it records as the $i$-th component of current state depending on whether or not station $i$ has a packet to transmit. As will become clear, with this state definition one can easily obtain the distribution of cycle time once the steady-state probabilities are computed.

State transition can be determined by examining the activities at each station in isolation, since the arrival processes at different stations are assumed to be independent. Specifically, for station $i$ we have

\begin{tabular}{c|c}
$n_{i} \rightarrow n_{i}^{\prime}$ & transition probability \\
\hline $0 \rightarrow 0$ & $e^{-\lambda_{i}(N W+S m)}$ \\
$0 \rightarrow 1$ & $1-e^{-\lambda_{i}(N W+S m)}$ \\
$1 \rightarrow 0$ & $e^{-\lambda_{i}(N W+S m)}$ \\
$1 \rightarrow 1$ & $1-e^{-\lambda_{i}(N W+S m)}$ \\
\hline
\end{tabular}

where $n_{i}^{\prime}$ is the next state of station $i . W$ is the token walk time from station to station, which consists of two components: the propagation and transmission delay from station to station, and the bit delay (which is associated with the station's relay delay and latency 
buffer delay) for a station, and $m$ is the time required for a packet to be transmitted. Under the assumption ${ }^{1}$ that the stations with a full buffer are uniformly distributed on the ring over the long run, $S=\sum_{i=1}^{N} n_{i}$ represents the number of stations with outstanding packets, and $N W+S m$ is the time the token has been absent from station $i$ since its last visit, i.e., the cycle time. Note that the influence of other station buffer occupancies on station $i$ is accounted for in the parameter $S$. The state transition probability can thus be expressed as

$$
p_{\vec{n} \rightarrow \vec{n}^{\prime}}=\prod_{i=1}^{N}\left(1-e^{-\lambda_{i}(N W+S m)}\right)^{n_{i}^{\prime}} \cdot\left(e^{-\lambda_{i}(N W+S m)}\right)^{1-n_{i}^{\prime}} .
$$

Note that this is not a Bernoulli trial but a Markov chain, since $S=\sum_{i=1}^{N} n_{i}$ depends on $\vec{n}$.

The state space of a homogeneous system can be reduced by defining $S$ - the number of occupied stations seen by the token as it cycles through all stations - as the state. The state transition probability then becomes

$$
p_{S, S^{\prime}}=\left(\begin{array}{c}
N \\
S^{\prime}
\end{array}\right)\left(1-e^{-\lambda(N W+S m)}\right)^{S^{\prime}} \cdot\left(e^{-\lambda(N W+S m)}\right)^{N-S^{\prime}}
$$

where $S^{\prime}=\sum_{i=1}^{N} n_{i}^{\prime}$.

\subsubsection{Cycle Time Distribution}

Let $\pi_{\vec{n}}\left(\pi_{S}\right)$ be the steady-state probability that the system is in state $\vec{n}$ (state $S$ ). Determination of the equilibrium probability distributions, $\pi_{\vec{n}}$, for all $\vec{n}$ ( $\pi_{s}$ for $0 \leq S \leq$ $N$ ), is straightforward (e.g., see [9] for a standard technique). The distribution of cycle time is

$$
P\left(T_{c}=N W+S m\right)=\sum_{\vec{n}: S=\sum_{j=1}^{N} n_{j}} \pi_{\vec{n}}=\pi_{S} .
$$

\subsubsection{Access Time Distribution}

The access delay at station $i$, denoted by $D_{A_{i}}$, can now be derived. The following derivation draws on the approach taken in [8]. Let $A_{i}$ denote the event of a packet arriving at station $i$ in a cycle. The joint probability of events $A_{i}$ and $T_{c}=N W+k m$ (i.e., $k$ packets in a cycle) is:

$$
P\left(T_{c}=N W+k m, A_{i}\right)=P\left(T_{c}=N W+k m\right) P\left(A_{i} \mid T_{c}=N W+k m\right) .
$$

The probability $P\left(T_{c}=N W+k m\right)$ is given by Eq. (3). The term $P\left(A_{i} \mid T_{c}=\right.$ $N W+k m)$ can be expressed as

$$
P\left(A_{i} \mid T_{c}=N W+k m\right)=\frac{\lambda_{i} \cdot \sum_{j_{1}, j_{2}, \ldots, j_{k-1} \in P W_{k-1}^{N-i}} \lambda_{j_{1}} \lambda_{j_{2}} \ldots \lambda_{j_{k-1}}}{\sum_{\ell_{1}, \ell_{2}, \ldots, \ell_{k} \in P W_{k}^{N-0}} \lambda_{\ell_{1}} \lambda_{\ell_{2}} \ldots \lambda_{\ell_{k}}},
$$


where $P W_{a}^{b-i}$ is the set of $a(<b)$ integers chosen from $\{\ell: 1 \leq \ell \leq b, \ell \neq$ $i, \ell$ is an integer $\}$. In case of homogeneous systems (e.g., $\lambda_{i}=\lambda, \forall i$ )

$$
P\left(A_{i} \mid T_{c}=N W+k m\right)=\frac{C_{k-1}^{N-1}}{C_{k}^{N}}=\frac{k}{N} .
$$

The cycle time conditioned on a packet arrival can be determined by using the above two equations and the property of total probability,

$$
P\left(T_{c}=N W+k m \mid A_{i}\right)=\frac{P\left(T_{c}=N W+k m, A_{i}\right)}{\sum_{k=1}^{N} P\left(T_{c}=N W+k m, A_{i}\right)} .
$$

By conditioning upon event $A_{i}$ and the duration of a cycle $T_{c}=N W+k m$, and by defining the time elapsed from the beginning of the cycle to the packet's arrival as $\tau=T_{2}-T_{1}-D_{A_{i}}=N W+(k-1) m-D_{A_{i}}$ (see Fig. 1), we now have

$$
\begin{aligned}
P\left(D_{A_{i}} \leq d \mid T_{c}=N W+k m, A_{i}\right) & =P\left(\tau \geq N W+(k-1) m-d \mid T_{c}=N W+k m, A_{i}\right) \\
& =1-P\left(\tau \leq N W+(k-1) m-d \mid T_{c}=N W+k m, A_{i}\right) .
\end{aligned}
$$

Note that the event $\left\{T_{c}=N W+k m, A_{i}\right\}$ is equivalent to $\left\{T_{c}=N W+k m, \tau \leq\right.$ $N W+(k-1) m\}$ since the event of a new packet arrival during a cycle implies that the time elapsed from the beginning of the cycle to the packet's arrival must be at least $m$ units of time less than the cycle time. Similarly, the event $\left\{T_{c}=N W+k m, A_{i}, \tau \leq\right.$ $N W+(k-1) m-d\}$ is equivalent to $\left\{\tau \leq N W+(k-1) m-d, T_{c}=N W+k m\right\}$, where $d$ is the access delay. Thus, we have

$$
\begin{aligned}
P\left(D_{A_{i}} \leq d \mid T_{c}=N W+k m, A_{i}\right) & =1-\frac{P\left(\tau \leq N W+(k-1) m-d, T_{c}=N W+k m\right)}{P\left(\tau \leq N W+(k-1) m, T_{c}=N W+k m\right)} \\
& =1-\frac{P\left(\tau \leq N W+(k-1) m-d \mid T_{c}=N W+k m\right)}{P\left(\tau \leq N W+(k-1) m \mid T_{c}=N W+k m\right)} \\
& =1-\frac{1-e^{-\lambda_{i}[N W+(k-1) m-d]}}{1-e^{-\lambda_{i}[N W+(k-1) m]}}=\frac{e^{\lambda_{i} d}-1}{e^{\lambda_{i}[N W+(k-1) m]}-1} .
\end{aligned}
$$

The second equality is obtained by dividing both the numerator and the denominator by $P\left(T_{c}=N W+k m\right)$. The third equality follows from the fact that the time to the first arrival is exponentially distributed and is independent of the cycle time, i.e., $P\left(\tau \leq N W+(k-1) m \mid T_{c}=N W+k m\right)=1-e^{-\lambda[N W+(k-1) m]}$. The unconditioned distribution of $D_{A_{i}}$ is then computed by averaging over $k$ as:

$$
\begin{aligned}
D_{A_{i}}(t) & \triangleq P\left(D_{A_{i}} \leq t \mid A_{i}\right) \\
& =\sum_{k} \frac{P\left(D_{A_{i}} \leq t, T_{c}=N W+k m, A_{i}\right)}{P\left(T_{c}=N W+k m, A_{i}\right)} \cdot \frac{P\left(T_{c}=N W+k m, A_{i}\right)}{P\left(A_{i}\right)} \\
& =\sum_{k} P\left(D_{A_{i}} \leq t \mid T_{c}=N W+k m, A_{i}\right) \cdot P\left(T_{c}=N W+k m \mid A_{i}\right) .
\end{aligned}
$$

Note that in the above summation, one must be careful in choosing the proper value of $t$, since for $t \geq N W+(k-1) m, P\left(D_{A_{i}} \leq t \mid T_{c}=N W+k m, A_{i}\right)=1$. Let $n(t)$ 


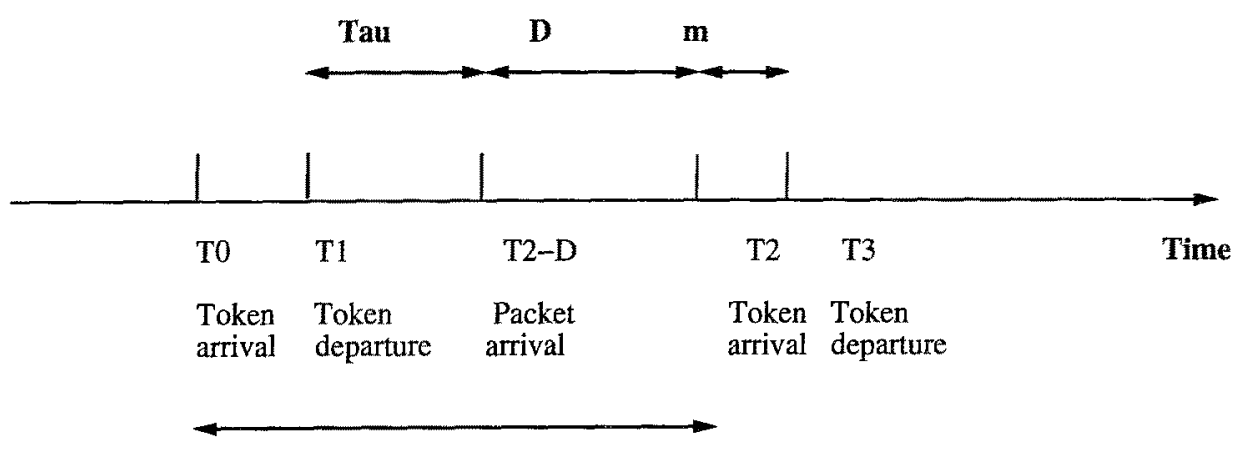

\section{Cycle time}

Figure 1. A timing diagram showing the access delay.

represent the maximum value of $n$ such that $n \leq \frac{t-N W}{m}+1$, and the above equation is reduced to

$$
\begin{aligned}
D_{A_{i}}(t) & =\sum_{k=1}^{n(t)} P\left(T_{c}=N W+k m \mid A_{i}\right) \\
& +\sum_{k=n(t)+1}^{N} P\left(D_{A_{i}} \leq t \mid T_{c}=N W+k m, A_{i}\right) P\left(T_{c}=N W+k m \mid A_{i}\right) .
\end{aligned}
$$

\subsubsection{Probability of Packet Rejection}

The probability of rejecting a packet is the same as that of a packet finding the buffer full upon its arrival. One may be tempted to express the probability of packet rejection as $Q_{i}^{*}=\sum_{\vec{n}: n_{i}=1} \pi_{\vec{n}}$. This is incorrect because the embedding point of the Markov chain to derive $\pi_{\vec{n}}$ is not at some arbitrary time instant, but as the token cycles through all stations. Thus, $Q_{i}^{*}$ represents the probability of a full buffer seen by the token as it visits station $i$, rather than that seen by a packet arriving at station $i$.

To derive the probability of packet rejection, each station is considered as an $\mathrm{M} / \mathrm{G} / 1 / 1$ queue. That is, packets arrive at station $i$ according to a Poisson distribution with rate $\lambda_{i}$, and are served by a single server with the distribution of service time:

$$
B_{i}(t)= \begin{cases}D_{A_{i}}(t-m) & \text { if } t \geq m \\ 0 & \text { otherwise. }\end{cases}
$$

The queue-length distribution for $\mathrm{M} / \mathrm{G} / 1 / 1$ queues has been well established (for example, see [7]), and the steady-state probability of a busy server (i.e., a full buffer in our case) at an arbitrary time instant has been shown to be $q_{1}^{i}=\frac{\lambda_{i}}{\lambda_{i}+\mu_{i}}$, where $\mu_{i}$ is the mean service rate (i.e., $\mu_{i}=\frac{1}{E\left(B_{i}(t)\right)}$ in our case). Besides, since the arrival process is 
Poisson, the general probability of a full buffer, $q_{1}^{i}$, is identical to the probability of a full buffer seen by an arriving packet at station $i$. Thus,

$$
Q_{i}=q_{1}^{i}=\frac{\lambda_{i} E\left(B_{i}(t)\right)}{\lambda_{i} E\left(B_{i}(t)\right)+1}
$$

\subsection{Token Ring Scheduling Protocol}

Under this protocol, a token with a reservation field is passed around the stations on the bus. Each station examines the reservation field as the token passes through and inserts the priority of its packet (if any) if and only if its priority is higher than the one currently in the reservation field. A station is allowed to capture the token and transmit its packet only when the token returns with the station's registered priority after passing through all the other stations. Consequently, the token has to come to the station with the highest-priority packet twice before this packet can be transmitted. The overhead thus incurred in each packet transmission is the ring walk time, $N W$. We include this overhead in the packet transmission time, which now becomes $N W+m$.

For the purpose of modeling, one can imagine that after the token comes to the station with the highest-priority packet, rather than traveling through all other stations to find that the station does have the highest-priority packet, it sits there for $N W$ units of time before starting transmission. Whether or not the token will stay at a station for $N W$ units of time during its visit is characterized by the probability of a station having the highest-priority packet (to be determined in Section 3.2.5). With this characterization, the cycle time is defined as the time required for the token to cycle through all stations once. Note that by 'once,' we exclude the time the token visits a station for polling, since the token is now viewed as staying in the station with the highest-priority packet during polling. (In reality, however, the token may visit a station more than once in each cycle time.)

\subsubsection{Definition and Transition of States}

The state of the system must contain: (1) buffer occupancy at each station, (2) the priority level of an outstanding packet, if any, at each station, and (3) the number of qualifiedto-transmit packets at a station as seen by the token. By 'qualified-to-transmit', we mean that the packet has the highest priority among all outstanding packets as the token comes to the station. The state is thus defined as $(\vec{n}, \vec{c})=\left(n_{1}, n_{2}, \ldots, n_{N} ; c_{1}, c_{2}, \ldots, c_{N}\right)$, where $n_{i}=1(0)$ if there is one (no) qualified-to-transmit packet as seen by the token, and $c_{i}$ is the priority of an outstanding packet at station $i\left(1 \leq c_{i} \leq p\right)$ as seen by the token. $c_{i} \triangleq 0$ when there is no packet at station $i$.

The transition probabilities for station $i$ are computed as follows: 


\begin{tabular}{c|c}
$\left(n_{i} ; c_{i}\right) \rightarrow\left(n_{i}^{\prime} ; c_{i}^{\prime}\right)$ & transition probability \\
\hline$(0 ; 0) \rightarrow(0 ; 0)$ & $e^{-\lambda_{i} C(S)}$ \\
$(0 ; 0) \rightarrow(1 ; j)$ & $\frac{\lambda_{i j}}{\lambda_{i}}\left(1-e^{-\lambda_{i} C(S)}\right) p_{i j}$ \\
$(0 ; 0) \rightarrow(0 ; j)$ & $\frac{\lambda_{i j}}{\lambda_{i}}\left(1-e^{-\lambda_{i} C(S)}\right)\left(1-p_{i j}\right)$ \\
$(0 ; j) \rightarrow(0 ; j)$ & $1-p_{i j}$ \\
$(0 ; j) \rightarrow(1 ; j)$ & $p_{i j}$ \\
$(1 ; j) \rightarrow(0 ; 0)$ & $e^{-\lambda_{i} C(S)}$ \\
$(1 ; j) \rightarrow(0 ; k)$ & $\frac{\lambda_{i k}}{\lambda_{i}}\left(1-e^{-\lambda_{i} C(S)}\right)\left(1-p_{i k}\right)$ \\
$(1 ; j) \rightarrow(1 ; k)$ & $\frac{\lambda_{i k}}{\lambda_{i}}\left(1-e^{-\lambda_{i} C(S)}\right) p_{i k}$ \\
\hline
\end{tabular}

where $1 \leq j, k \leq p, S=\sum_{i=1}^{N} n_{i}$ is the number of qualified-to-transmit packets seen by the token as it cycles through all stations, $C(S) \triangleq N W+S(m+N W)$ is the cycle time, and $p_{i j}$ is the probability that a priority $-j$ packet at station $i$ has the highest priority (as the token "cycles" through the ring), which is yet to be determined (Section 3.2.3). Derivation of these transition probabilities is straightforward; e.g., $\frac{\lambda_{i k}}{\lambda_{i}}\left(1-e^{-\lambda_{i} C(S)}\right)$ is the probability that there is an arrival of priority $k$ within the cycle time $C(S)$.

An implicit assumption used in deriving these transition probabilities is that a priority$k(<j)$ packet arriving at a downstream station (relative to the token's current position) during the polling time of a priority- $j$ packet is not allowed to abort the polling. In other words, if a priority $-j$ packet has the highest priority among all currently outstanding packets and initiates polling, it will surely gain medium access at the end of polling, regardless whether or not there will be subsequent arrivals of higher-priority packets within $N W$. This approximation does not sway the analytic results from reality when $N W<<m$ in which case the probability of new higher-priority packet arrivals within $N W$ is usually negligible. When $N W \approx m$, the results so derived for high-priority packets are worse than the simulations results, and may serve as a lower bound of performance. The state transition probabilities can be expressed in a way similar to the token passing protocol (Eq. (3.1)).

The state space can be reduced by using the homogeneity assumption. For example, if there are only two priority levels ${ }^{2}$ at station $i$ with arrival rates $\lambda_{i 1}$ and $\lambda_{i 2}$, respectively, we may define the state as $\left(s_{1} ; s_{2}, t_{2}\right)$, where $s_{1}, s_{2}$, and $t_{2}$ are the number of qualified-to-transmit high-priority packets, the number of qualified-to-transmit lowpriority packets, and the number of outstanding low-priority packets, all of which are seen by the token as it "cycles" through all stations. ${ }^{3}$ Note that with this definition of state, we have $0 \leq s_{2} \leq t_{2} \leq N, 0 \leq s_{1}+t_{2} \leq N$, and the size of the state space is now $\frac{(N+1)(N+2)(N+3)}{6}$.

Let $q_{1}(S)=\frac{\lambda_{i 1}}{\lambda_{i}}\left(1-e^{-\lambda_{i} C(S)}\right)\left(q_{2}(S)=\frac{\lambda_{i 2}}{\lambda_{i}}\left(1-e^{-\lambda_{i} C(S)}\right)\right)$ denote the probability that a high-priority (low-priority) packet arrives within a cycle time of $C(S)$, and $p_{2} \triangleq p_{i 2}$ $\forall i$, then the state transition probability can be expressed, under the assumption of statistically identical behavior of all stations, as:

$$
p_{\left(s_{1} ; s_{2}, t_{2}\right) \rightarrow\left(s_{1}^{\prime} ; s_{2}^{\prime}, t_{2}^{\prime}\right)}=
$$




$$
\begin{aligned}
& \left\{\frac{\left(N-\left(t_{2}-s_{2}\right)\right) !}{s_{1}^{\prime} !\left[t_{2}^{\prime}-\left(t_{2}-s_{2}\right)\right] !\left(N-s_{1}^{\prime}-t_{2}^{\prime}\right) !} \cdot\left[q_{1}\left(s_{1}+s_{2}\right)\right]^{s_{1}^{\prime}} \cdot\left[q_{2}\left(s_{1}+s_{2}\right)\right]^{t_{2}^{\prime}-\left(t_{2}-s_{2}\right)}\right. \\
& \left.\left[1-q_{1}\left(s_{1}+s_{2}\right)-q_{2}\left(s_{1}+s_{2}\right)\right]^{N-s_{1}^{\prime}-t_{2}^{\prime}}\right\} \cdot\left(\begin{array}{c}
t_{2}^{\prime} \\
s_{2}^{\prime}
\end{array}\right) p_{2}^{s_{2}^{\prime}}\left(1-p_{2}\right)^{t_{2}^{\prime}-s_{2}^{\prime}}
\end{aligned}
$$

Note that the term inside the braces is the probability of $s_{1}^{\prime}$ new arrivals of high-priority packets and $t_{2}^{\prime}-\left(t_{2}-s_{2}\right) \geq 0$ new arrivals of low-priority packets ${ }^{4}$ among $N-\left(t_{2}-s_{2}\right)$ stations with no outstanding packets. The term after the braces is the probability that $s_{2}^{\prime}$ out of $t_{2}^{\prime}$ low-priority packets find no high-priority packets waiting in the system as the token comes to their stations.

\subsubsection{Cycle Time Distribution}

A standard technique can be applied to obtain equilibrium probability distributions $\left(\pi_{(\vec{n}, \vec{c})}\right.$ or $\left.\pi_{\left(s_{1} ; s_{2}, t_{2}\right)}\right)$. The probability density function of $T_{c}$ can be written as

$$
P\left(T_{c}=N W+S(m+N W)\right)=\sum_{(\vec{n}, \vec{c}): \sum_{j=1}^{N} n_{j}=S} \pi_{(\vec{n}, \vec{c})}
$$

or

$$
P\left(T_{c}=N W+S(m+N W)\right)=\sum_{\left(s_{1} ; s_{2}, t_{2}\right): s_{1}+s_{2}=S} \pi_{\left(s_{1} ; s_{2}, t_{2}\right)}
$$

\subsubsection{Access Delay Distribution}

To derive the distribution of the access delay, we follow the same approach as in the token passing protocol except that (1) $N W+k m$ has to be replaced by $N W+k(m+N W)$, (2) the counterpart of $P\left(A_{i} \mid T_{c}=N W+k m\right)$ in Eq. (5), i.e., $P\left(A_{i} \mid T_{c}=N W+\right.$ $k(m+N W))$, must be approximated, since given $T_{c}$ (or, equivalently $k$ ) there is no information about the number of packets that have arrived during the cycle time. ${ }^{5}$ Two cases are considered; (1) a packet of priority $j$ arrives and turns out to have the highest priority among all outstanding packets as the token comes to it; (2) a packet of priority $j$ arrives and does not have the highest priority as the token comes to it.

Let $A_{i}^{<j>}$ denote the event of a priority-j packet arriving at station $i$ in a cycle. Then in the first case,

$$
P\left(A_{i}^{<j>} \mid T_{c}=N W+k(m+N W)\right)=\frac{\lambda_{i j}}{\lambda_{i}}\left(1-e^{-(N W+(k-1)(N W+m)) \lambda_{i}}\right),
$$

since given that the priority $-j$ packet arriving during this cycle was transmitted upon the token's first visit, it must have arrived in last $N W+(k-1)(m+N W)$ units of time (see Fig. 1). Let $D_{A_{i 1}}^{<j>}$ denote the time between the arrival of such a packet and the token's visit. Then, all the subsequent derivation for $P\left(D_{A_{i 1}}^{<j>} \leq t\right)$ is the same as that for $P\left(D_{A_{i}} \leq t\right)$ in the token passing protocol. 
In the second case, a priority- $j$ packet arriving at a station in one cycle is not transmitted when the token visits the station (i.e., it does not contribute $m+N W$ units of time to the cycle time), and thus could join the system in $N W+k(m+N W)$ units of time. In other words,

$$
P\left(A_{i}^{<j>} \mid T_{c}=N W+k(m+N W)\right)=\frac{\lambda_{i j}}{\lambda_{i}}\left(1-e^{-(N W+k(m+N W)) \lambda_{i}}\right) .
$$

Furthermore, for the second case the event $\left\{T_{c}=N W+k(m+N W), A_{i}^{<j>}\right\}$ is equivalent to $\left\{T_{c}=N W+k(m+N W), \tau \leq N W+k(m+N W)\right\}$ and the event $\left\{T_{c}=N W+k(N W+m), A_{i}^{<j>}, \tau \leq N W+k(m+N W)-d\right\}$ is equivalent to $\left\{\tau \leq N W+k(m+N W)-d, T_{c}=N W+k(m+N W)\right\}$. Consequently, if $D_{A_{i 2}}^{<j>}$ denotes the time between the arrival of such a packet and the token's first visit since this arrival, we have

$$
\begin{aligned}
& P\left(D_{A_{i 2}}^{<j>} \leq d \mid T_{c}=N W+k(m+N W), A_{i}\right) \\
& =1-\frac{P\left(\tau \leq N W+k(m+N W)-d, T_{c}=N W+k(m+N W)\right)}{P\left(\tau \leq N W+k(m+N W), T_{c}=N W+k(m+N W)\right)} \\
& =1-\frac{\frac{\lambda_{i j}}{\lambda_{i}}\left(1-e^{-\lambda_{i}[N W+k(m+N W)-d]}\right)}{\frac{\lambda_{i j}}{\lambda_{i}}\left(1-e^{-\lambda_{i}[N W+k(m+N W)]}\right)}=\frac{e^{\lambda_{i} d}-1}{e^{\lambda_{i}[N W+k(m+N W)]}-1} .
\end{aligned}
$$

All the subsequent derivation for $P\left(D_{A_{i 2}}^{<j>} \leq t\right)$ is similar to that for $P\left(D_{A_{i}} \leq t\right)$ in the case of token passing protocol.

The distribution of the access delay of a priority- $j$ packet, $P\left(D_{A_{i}}^{<j>} \leq t\right)$, can be approximately derived by considering whether or not the arriving packet of priority $j$ has the highest priority among all outstanding packets (and is thus transmitted upon the token's visit). That is,

$$
P\left(D_{A_{i}}^{<j>} \leq t\right)=p_{i j} P\left(D_{A_{i 1}}^{<j>} \leq t\right)+\sum_{k=1}^{\infty}\left(1-p_{i j}\right)^{k} p_{i j} P\left(D_{A_{i 2}}^{<j>} \leq t\right) * P^{(k)}\left(T_{c} \leq t\right),
$$

where $P^{(k)}\left(T_{c} \leq t\right)$ denotes the distribution of the $(k-1)$-fold convolution of the density function of $T_{c}$, and the symbol * denotes the convolution performed on the density functions of the two probability distributions of interest.

\subsubsection{Probability of Packet Rejection}

To derive the probability of packet rejection, each station is (again) modeled as an $\mathrm{M} / \mathrm{G} / 1 / 1$ queue with the service time distribution

$$
B_{i}(t)= \begin{cases}\sum_{j=1}^{p} \frac{\lambda_{i j}}{\lambda_{i}} D_{A_{i}}^{<j>}(t-(m+N W)) & \text { if } t \geq m+N W \\ 0 & \text { otherwise. }\end{cases}
$$

Then, using the same arguments as in the token passing protocol (Section 3.1.4), $Q_{i}$ can be shown to be $Q_{i}=\frac{\lambda_{i} E\left(B_{i}(t)\right)}{\lambda_{i} E\left(B_{i}(t)\right)+1}$. 


\subsubsection{Determination of $p_{i j}$}

To derive $p_{i j}$ - the probability that a priority $-j$ packet in station $i$ has the highest priority among all outstanding packets - we have to construct another Markov chain embedded in system evolution. Define the time interval between two successive packet transmissions as period, and examine the system state at the end of each period. The state is defined as $\vec{r}=\left(r_{1}, r_{2}, \ldots, r_{N}\right)$ where $r_{i}$ is the priority of the outstanding packet at station $i .1 \leq r_{i} \leq p$, and $r_{i} \triangleq 0$ if there is no outstanding packet at station $i$.

Note that each period consists of the following three subintervals: (1) polling interval which equals $N W$, and the time for the token to poll all the other stations before returning to the station with the highest-priority packet; (2) transmission interval which follows the polling interval and equals $m$. (3) idle interval which is the time interval between the end of the transmission interval and the beginning of the next polling interval.

There will be a nonzero idle interval if the highest-priority packet is not located at the immediate neighbor station in the downstream, and can be expressed as $K_{\text {idle }} W$, where $K_{i d l e}$ is a positive integer random variable. The derivation of the exact distribution of $K_{\text {idle }}$ depends on (i) when and where a packet will arrive if there is no packet waiting in the system after a transmission interval, or (ii) the location of the highest-priority packet if there is at least one packet waiting in the system, or (iii) whether or not a higherpriority packet will arrive during the polling initiated by a station holding a packet, and thus terminate the on-going polling. Moreover, if this occurs, the derivation also depends on the location of this newly arriving packet.

Dynamically-changing conditions with the state of the system make it very difficult to derive the exact distribution of $K_{\text {idle }}$. So, we approximate this distribution as follows. Except for those systems with very light traffic, it is reasonable to assume the existence of at least one outstanding packet in the system, thus excluding the possibility of condition (i). When $N W<<m$, or, when there are not many priority levels, the effect of condition (iii) on $K_{\text {idle }}$ is minimal, and thus we assume that a polling, once it begins, is never aborted. Hence, $P\left(K_{\text {idle }} \leq N\right) \approx 1$ will hold for all but those systems with very light traffic. Moreover, under the assumption of the statistically identical behavior of all stations, each station in the downstream (relative to the token's current position) is equally likely to have the highest-priority packet. Thus, one can approximate the distribution of $K_{\text {idle }}$ to be uniformly distributed over $[1, N]$. (The data gathered from our simulations validate this approximate distribution.) The length of each period is thus equal to $m+N W+K_{i d l e} W$.

Now, define $\mu_{i}(\vec{r})=0(1)$ if $r_{i}>(=) \min \left(r_{1}, \ldots, r_{N}\right)$ as the indicator variable that station $i$ holds a high-priority packet, and let $\nu(\vec{r})=\sum_{i=1}^{N} \mu_{i}(\vec{r})$ be the number of stations with currently highest-priority packets, then the transition probabilities for station $i$ can be approximated as:

\begin{tabular}{c|c}
$r_{i} \rightarrow r_{i}^{\prime}$ & transition probability \\
\hline$j \rightarrow j$ & $1-\frac{1}{\nu(\bar{r})} \mu_{i}(\vec{r})$ \\
$j \rightarrow 0$ & $\frac{1}{\nu(\vec{r})} \mu_{i}(\vec{r})$ \\
$0 \rightarrow 0$ & $E\left(e^{\lambda_{i}\left(m+N W+K_{i d l e} W\right)}\right)$ \\
$0 \rightarrow j$ & $\frac{\lambda_{i j}}{\lambda_{i}}\left(1-E\left(e^{\lambda_{i}\left(m+N W+K_{i d l e} W\right)}\right)\right)$ \\
\hline
\end{tabular}


where $1 \leq j \leq p$, and the expectation $E(\cdot)$ is taken with respect to $K_{\text {idle. Note }}$. Nhat for ease of analysis, we exclude, in the calculation of transition probability $0 \rightarrow 0$, the possibility that a packet arriving at an idle station will find no other outstanding packet with a priority higher than itself and will thus get transmitted immediately upon its arrival. This possibility is unlikely to exist for all but the case of very light traffic, and the resulting discrepancy is shown (through simulations) to be tolerably small.

The state transition probabilities can be obtained using the standard technique. Let $\phi_{\vec{r}}$ be the equilibrium probability that the system is in state $\vec{r}$. Then,

$$
p_{i j}=\frac{\sum_{\vec{r}: r_{i}=j, \text { and } r_{i}=\min \left(r_{1}, r_{2}, \ldots, r_{N}\right)} \phi_{\vec{r}}}{\sum_{\vec{r}: r_{i}=j} \phi_{\vec{r}}} .
$$

\subsection{The $P_{i}$-Persistent Protocol}

In the $P_{i}$-persistent protocol, time is slotted with a slot equal to the packet transmission time, $m .^{6}$ Each station monitors the channel constantly, and persists to transmit a packet (if any) in each idle slot with probability $p_{i}$. If the station initially senses the medium busy, it waits until the next slot, and if the medium becomes idle in the next slot, the station transmits with a probability $p_{i}$ or defers with a probability $1-p_{i}$.

\subsubsection{State Definition \& Transition, and Parameter Derivation}

A station's state at the end of a slot $k$ depends only on its state at the end of slot $k-1$ and the activity (packet arrival and/or transmission) during slot $k$. Thus, we may focus on each station's buffer occupancy at the end of a slot. That is, the state of station $i, 1 \leq i \leq N$, is defined as $n_{i}$, where $n_{i} \in\{0,1\}$ is the buffer occupancy at the end of each slot. The transition probabilities can be expressed as a function of $c_{i} \triangleq P$ (slot seen by station $i$ is empty) (which will be determined below):

\begin{tabular}{c|c}
$n_{i} \rightarrow n_{i}^{\prime}$ & transition probability \\
\hline $0 \rightarrow 0$ & $e^{-\lambda_{i} m}$ \\
$0 \rightarrow 1$ & $1-e^{-\lambda_{i} m}$ \\
$1 \rightarrow 0$ & $p_{i} c_{i} e^{-\lambda_{i} m}$ \\
$1 \rightarrow 1$ & $1-p_{i} c_{i} e^{-\lambda_{i} m}$ \\
\hline
\end{tabular}

Station $i$ 's equilibrium probability distribution $\left\{\pi_{i j}, j=0,1\right\}$ can be determined easily: for $1 \leq i \leq N$,

$$
\pi_{i 1}=\frac{1-e^{-\lambda_{i} m}}{1-e^{-\lambda_{i} m}+p_{i} c_{i} e^{-\lambda_{i} m}}, \quad \pi_{i 0}=\frac{p_{i} c_{i} e^{-\lambda_{i} m}}{1-e^{-\lambda_{i} m}+p_{i} c_{i} e^{-\lambda_{i} m}} .
$$

$c_{i}$ can then be expressed as: for $1 \leq i \leq N$

$c_{i}=P$ (stations other than station $i$ do not transmit) 


$$
\begin{aligned}
= & \prod_{k=1, k \neq i}^{N} \pi_{k 0}+\sum_{k=1, k \neq i}^{N} \pi_{k 1}\left(1-p_{k}\right) \prod_{j=1, j \neq i, k}^{N} \pi_{j 0} \\
& +\sum_{k, \ell \in P W_{2}^{N-i}} \pi_{k 1}\left(1-p_{k}\right) \pi_{\ell 1}\left(1-p_{\ell}\right) \prod_{j=1, j \neq i, k, \ell}^{N} \pi_{j 0}+\ldots+\prod_{k=1, k \neq i}^{N} \pi_{k 1}(1-p(k) 8)
\end{aligned}
$$

where $P W_{2}^{N-i}$ is the set of two integers chosen from $\{a: 1 \leq a \leq N, a \neq i, a$ is an integer $\}$. $\mathrm{Eq}$. (18) together with $\mathrm{Eq}$. (17) can be used to solve $c_{i}$ for $1 \leq i \leq N$ (2N equations with $2 N$ unknowns). The assumption of system homogeneity, or $p_{i}=p, \lambda_{i}=\lambda$, and $\pi_{i 1}=\pi_{1}, \forall i$, can simplify the expression for $c_{i}=c$ to:

$$
c=\sum_{i=0}^{N-1}\left(\begin{array}{c}
N-1 \\
i
\end{array}\right) \pi_{1}^{i}(1-p)^{i} \pi_{0}^{N-1-i}=\left(1-\pi_{1} p\right)^{N-1} .
$$

One can solve Eq. (19) (along with Eq. (17)) numerically to get $c$. The access delay is then given by

$$
D_{A_{i}}(t)=\sum_{k=0}^{\lfloor t / m\rfloor}\left(1-c_{i} p_{i}\right)^{k} c_{i} p_{i}
$$

Note that arrival of a packet may not be synchronized to a slot boundary, and thus, an additional synchronization time $T_{s} \in(0,1)$ should be added to the access delay. However, $T_{s}$ has been omitted from our model for the following reasons: (1) $E\left(T_{s}\right)$ 's are the same for all stations in a homogeneous system, (2) except for light traffic cases, the delay due to the contention and collisions is much larger than one slot period, and thus, omitting $T_{s}$ may introduce an error of at most one slot, which is tolerably small.

The expression of the probability of packet rejection, $Q_{i}$, is the same as that in the token passing protocol (Section 3.1.4).

\subsection{Priority-Based $P_{i}-$ Persistent Protocol}

In the simple priority-based variation of $P_{i}$-persistent protocol, each station persists to transmit a priority $-j$ packet in a slot with probability $p_{i j}$. The higher the priority $j$, the larger the persistence probability $p_{i j}$. The approach taken to analyze the $P_{i}$-persistent protocol can be applied directly to this protocol except that the state of station $i$ is now defined as $r_{i}$, where $r_{i}$ is the priority of the outstanding packet, if any, at station $i\left(1 \leq r_{i} \leq p\right)$; otherwise, $r_{i}=0$. The transition probabilities for station $i$ can be expressed as:

\begin{tabular}{c|c}
$r_{i} \rightarrow r_{i}^{\prime}$ & transition probability \\
\hline $0 \rightarrow 0$ & $e^{-\lambda_{i} m}$ \\
$0 \rightarrow j$ & $\frac{\lambda_{i j}}{\lambda_{i}}\left(\mathbf{1}-e^{-\lambda_{i} m}\right)$ \\
$j \rightarrow 0$ & $p_{i j} c_{i} e^{-\lambda_{i} m}$ \\
$j \rightarrow k$ & $p_{i j} c_{i} \frac{\lambda_{i k}}{\lambda_{i}}\left(1-e^{-\lambda_{i} m}\right)$ \\
$j \rightarrow j$ & $1-p_{i j} c_{i}+p_{i j} c_{i} \frac{\lambda_{i j}}{\lambda_{i}}\left(1-e^{-\lambda_{i} m}\right)$ \\
\hline
\end{tabular}


where $1 \leq j, k \leq p$. The equilibrium probability distributions, $\left\{\pi_{i j}, 1 \leq j \leq p\right\}$, can be derived in terms of $c_{i}$, which can in turn be expressed as a function of $\pi_{k j}$ 's, $1 \leq k \leq N, k \neq i, 1 \leq j \leq p$. Thus, one can, in principle, solve for both $r_{i}$ 's and $\pi_{i j}$ 's. By the assumption of system homogeneity $\left(p_{i j}=p_{j}, c_{i}=c\right.$, and $\pi_{i j}=\pi_{j}$, for all $i$ ) and the assumption of two-priority levels ${ }^{7}$ (each having arrival rate $\lambda_{i 1}$ and $\lambda_{i 2}$ at a station), we have

$$
\begin{aligned}
& \pi_{1}=\frac{\frac{\lambda_{i 1}}{\lambda_{i}} c p_{2}\left(1-e^{-\lambda m}\right)}{p_{1} p_{2} c^{2} e^{-\lambda_{i} m}+c\left(\frac{\lambda_{i 1} 1}{\lambda_{i}} p_{2}+\frac{\lambda_{i 2} p_{1}}{\lambda_{i}} p_{1}\left(1-e^{-\lambda_{i} m}\right)\right.}, \\
& \pi_{2}=\frac{\frac{\lambda_{i 2}}{\lambda_{i}} c p_{1}\left(1-e^{-\lambda_{i} m}\right)}{p_{1} p_{2} c^{2} e^{-\lambda_{i} m}+c\left(\frac{\lambda_{i 1}}{\lambda_{i}} p_{2}+\frac{\lambda_{i 2}}{\lambda_{i}} p_{1}\right)\left(1-e^{-\lambda_{i} m}\right)},
\end{aligned}
$$

and

$$
c=\left(1-\pi_{1} p_{1}-\pi_{2} p_{2}\right)^{N-1} .
$$

Eq. (22) together with Eq. (21) can be solved numerically to determine $c$. The access delay for a priority $-j$ packet is then given by

$$
D_{A_{i}}^{<j>}(t)=\sum_{k=0}^{\lfloor t / m\rfloor}\left(1-c_{i} p_{i j}\right)^{k} c_{i} p_{i j} .
$$

The approach to derive $Q_{i}$ is the same as that for the token scheduling protocol.

\section{Extension to Multiple-Buffer Case}

Though the single-buffer model eases analysis and can be used to evaluate the goodness of a contention protocol, we need a multiple-buffer model to study the performance differences between the single and multiple buffer cases. To meet this need, we now extend the single-buffer model to the case of $L$ buffers in each station.

\subsection{Token Passing Protocol}

\subsubsection{Definition and Transition of States}

The embedded Markov chain constructed for the single-buffer model is also used here to describe the system except that $n_{i}\left(0 \leq n_{i} \leq L\right)$ in the state $\vec{n}=\left(n_{1}, n_{2}, \ldots, n_{N}\right)$ is now defined as the number of packets at station $i$ seen by the token as it cycles through all stations. The station transition probabilities can be determined by examining the activities at each station $i$, and are given by: 


$$
p_{n_{i} \rightarrow n_{i}^{\prime}}= \begin{cases}0 & \text { if } n_{i}^{\prime} \leq n_{i}-2 \\ \frac{e^{-(N W+S m) \lambda_{i}}\left[\lambda_{i}(N W+S m)\right]_{i}^{n_{i}^{\prime}}}{n_{i}^{\prime} !} & \text { if } n_{i}=0 \text { and } 0 \leq n_{i}^{\prime} \leq L-1 \\ 1-\sum_{k=0}^{L-1} \frac{e^{-(N W+S m) \lambda_{i}}\left[\lambda_{i}(N W+S m)\right]^{k}}{k !} & \text { if } n_{i}=0 \text { and } n_{i}^{\prime}=L \\ \frac{e^{-(N W+S m) \lambda_{i}}\left[\lambda_{i}(N W+S m)\right]^{\left(n_{i}^{\prime}-n_{i}+1\right)}}{\left(n_{i}^{\prime}-n_{i}+1\right) !} & \text { if } n_{i} \geq 1 \text { and } n_{i}-1 \leq n_{i}^{\prime} \leq L-1 \\ 1-\sum_{k=0}^{L-n_{i}} \frac{e^{-(N W+S m) \lambda_{i}}\left[\lambda_{i}(N W+S m)\right]^{k}}{k !} & \text { if } n_{i} \geq 1 \text { and } n_{i}^{\prime}=L .\end{cases}
$$

Under the assumption that the stations with a full buffer are uniformly distributed on the ring over the long run, $S=\sum_{j=1}^{N}\left(1-\delta\left(n_{j}\right)\right)$ denotes the number of stations with outstanding packets, where $\delta\left(n_{j}\right)=1$, for $n_{j}=0 ; 0$, otherwise. The dependence of $S$ on $\vec{n}$ accounts for the influence of other stations' buffer occupancy on station $i$ 's state transition.

Under the system homogeneity assumption, states can be aggregated, thus reducing the state space in a way similar to (but more complex than) the single-buffer model. Determination of the equilibrium probabilities, $\pi_{\vec{n}}$, is straightforward, and the distribution of cycle time can be expressed as

$$
P\left(T_{c}=N W+S m\right)=\sum_{\vec{n}: S=\sum_{j=1}^{N}\left(1-\delta\left(n_{j}\right)\right)} \pi_{\vec{n}}
$$

\subsubsection{Queue Length Distribution}

Having obtained the distribution of cycle time, we are now in a position to derive the distribution of queue length which will then be used to derive $Q_{i}$ and $D_{A_{i}}(t)$. Each station $i$ can be considered as an M/G/1/L queue, where the service time has the probability density function, $b_{i}(t)=T_{c}(t)$, and $T_{c}(t)$ is the probability density function of cycle time. The expression for $b_{i}(t)$ is exact when there is at least one outstanding packet at station $i$, but is only approximate when there is no outstanding packet at station $i$, since a packet arriving at an empty station $i$ has the service time cumulative distribution $B_{i}(t)=D_{A_{i 1}}(t-m)$, for $t \geq m ; 0$, otherwise, where $D_{A_{i 1}}$ is derived in a way similar to that in Section 3.2.3.

The length distribution of the $\mathrm{M} / \mathrm{G} / 1 / L$ queue, $\{q(j), 0 \leq j \leq L\}$, can be expressed in terms of the length distribution of the corresponding $\mathrm{M} / \mathrm{G} / 1 / \infty$ queue (for example, see [10]). That is, if $\{q(j), 0 \leq j \leq L\}$ and $\left\{q^{*}(j), j \geq 0\right\}$ represent the length distribution of the $\mathrm{M} / \mathrm{G} / 1 / L$ queue and the corresponding $\mathrm{M} / \mathrm{G} / 1 / \infty$ queue, respectively, then the following relation holds:

$$
q(j)=f q^{*}(j), \text { for } 0 \leq j \leq L-1, \quad \text { and } \quad q(L)=1-\frac{1-f q^{*}(0)}{\lambda E(B(t))},
$$

where $f=1 /\left(q^{*}(0)+\lambda E(B(t)) \sum_{j=0}^{L-1} q^{*}(j)\right), E(B(t))$ is the mean service time, and $\left\{q^{*}(j), j \geq 0\right\}$ can be obtained from the well-known Pollaczek-Khinchin transform equation [7], [10]. 


\subsubsection{Probability of Packet Rejection and Distribution of Access Delay}

Given the probability distribution, $\left\{q^{i}(j), 0 \leq j \leq L\right\}$, that there are $j$ packets at station $i$, the probability of packet rejection can be expressed as $Q_{i}=q^{i}(L)$. The distribution of access delay can be approximately expressed as

$$
P\left(D_{A i} \leq t\right)=q^{i}(0) P\left(D_{A_{i 1}} \leq t\right)+\sum_{k=1}^{L-1} q^{i}(k) P\left(D_{A_{i 2}} \leq t\right) * P^{(k)}\left(T_{c} \leq t\right),
$$

where $P^{(k)}\left(T_{c} \leq t\right)$ indicates the distribution of the $k$-fold convolution of the density function of $T_{c}$, the symbol $*$ denotes the convolution performed on the density functions of two probability distributions of interest, and $D_{A_{i 1}}(t)$ and $D_{A_{i 2}}(t)$ are derived in a similar way ${ }^{8}$ as in Section 3.2.3.

\subsection{Token Scheduling Protocol}

\subsubsection{Definition and Transition of States}

The state of station $i$ is defined as $\left(n_{i 1} ; n_{i 2}, n_{i 3}\right)$, where $n_{i 1}$ is the number of qualifiedto-transmit high-priority packets, $n_{i 2}=1(0)$ indicates the event that a low-priority packet is (not) qualified to transmit, and $n_{i 3}$ is the number of outstanding low-priority packets, all of which are seen by the token as it comes to station $i$. Here we again adopt the imaginary scenario in Section 3.2 to characterize/define the cycle time. State transition probabilities can be determined in a way similar to (but more complex than) the single-buffer model, and are given in Table 1. Note that $S=\sum_{j=1}^{N}\left[\left(1-\delta\left(n_{j 1}\right)\right)+n_{j 2}\right]$ in Table 1 is the number of qualified-to-transmit packets seen by the token as it "cycles" through all stations. The distribution of cycle time can then be expressed in terms of the equilibrium probabilities, $\pi_{\left(\overrightarrow{n_{1}} ; \overrightarrow{n_{2}}, \overrightarrow{n_{3}}\right)}$ :

$$
P\left(T_{c}=N W+S(m+N W)\right)=\sum_{\left(\overrightarrow{n_{1}} ; \overrightarrow{n_{2}}, \overrightarrow{n_{3}}\right): S=\sum_{j=1}^{N}\left[\left(1-\delta\left(n_{j 1}\right)\right)+n_{j 2}\right]} \pi_{\left(\overrightarrow{n_{1}} ; \overrightarrow{n_{2}}, \overrightarrow{n_{3}}\right)} .
$$


Table I. State transition probabilities for the token scheduling protocol. $C(s) \triangleq N W+s(m+N W)$ is the cycle time given that there are $s$ packets transmitted during the cycle, $p_{i 2}=\mathrm{P}$ (no high-priority packet waiting in the system as the token comes to station $i)$, and $S=\sum_{j=1}^{N}\left[\left(1-\delta\left(n_{j 1}\right)\right)+n_{j 2}\right]$.

\begin{tabular}{|c|c|}
\hline $\begin{array}{l}\left(n_{i 1} ; n_{i 2}, n_{i 3}\right) \\
\rightarrow\left(n_{i 1}^{\prime} ; n_{i 2}^{\prime}, n_{i 3}^{\prime}\right)\end{array}$ & $\begin{array}{l}\text { Transition probability } \\
\text { for station } i\end{array}$ \\
\hline \multirow[t]{2}{*}{$\begin{array}{l}\left(n_{i 1} ; 0, n_{i 3}\right) \\
\rightarrow\left(0 ; 1, n_{i 3}^{\prime}\right)\end{array}$} & $\begin{array}{r}e^{-\lambda_{i 1} C(s)} \cdot \frac{e^{-\lambda_{i 2} C(s)}\left(\lambda_{i 2} C(s)\right)^{\left(n_{i 3}^{\prime}-n_{i 3}\right)}}{\left(n_{i 3}^{\prime}-n_{i 3}\right) !} \cdot p_{i 2} \\
0 \leq n_{i 1} \leq 1, \max \left(1, n_{i 3}\right) \leq n_{i 3}^{\prime} \leq L_{2}-1\end{array}$ \\
\hline & $\begin{array}{l}e^{-\lambda_{i 1} C(s)} \cdot\left[1-\sum_{n_{i 3}^{\prime}=n_{i 3}}^{L_{2}-1} \frac{e^{-\lambda_{i 2} C(s)}\left(\lambda_{i 2} C(s)\right)^{\left(n_{i 3}^{\prime}-n_{i 3}\right)}}{\left(n_{i 3}^{\prime}-n_{i 3}\right) !}\right] p_{i 2}, \\
0 \leq n_{i 1} \leq 1, n_{i 3}^{\prime}=L_{2}\end{array}$ \\
\hline \multirow[t]{11}{*}{$\begin{array}{l}\left(n_{i 1} ; 0, n_{i 3}\right) \\
\rightarrow\left(n_{i 1}^{\prime} ; 0, n_{i 3}^{\prime}\right)\end{array}$} & $\begin{array}{l}e^{-\lambda_{i 1} C(s)} \cdot e^{-\lambda_{i 2} C(s)} \\
0 \leq n_{i 1} \leq 1, n_{i 1}^{\prime}=n_{i 3}=n_{i 3}^{\prime}=0\end{array}$ \\
\hline & 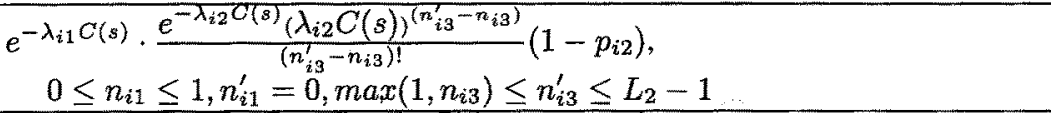 \\
\hline & $\begin{array}{l}e^{-\lambda_{i 1} C(s)} \cdot\left[1-\sum_{n_{i 3}^{\prime}=n_{i 3}}^{L_{2}-1} \frac{e^{-\lambda_{i 2} C(s)}\left(\lambda_{i 2} C(s)\right)^{\left(n_{i 3}^{\prime}-n_{i 3}\right)}}{\left(n_{i 3}^{\prime}-n_{i 3}\right) !}\right]\left(1-p_{i 2}\right) \\
\quad 0 \leq n_{i 1} \leq 1, n_{i 1}^{\prime}=0, n_{i 3}^{\prime}=L_{2}\end{array}$ \\
\hline & $\begin{array}{c}\frac{e^{-\lambda_{i 1} C(s)}\left(\lambda_{i 1} C(s)\right)^{n_{i 1}^{\prime}}}{n_{i 1}^{\prime} !} \cdot \frac{e^{-\lambda_{i 2} C(s)}\left(\lambda_{i 2} C(s)\right)^{\left(n_{i 3}^{\prime}-n_{i 3}\right)}}{\left(n_{i 3}^{\prime}-n_{i 3}\right) !} \\
n_{i 1}=0,1 \leq n_{i 1}^{\prime} \leq L_{1}-1, n_{i 3} \leq n_{i 3}^{\prime} \leq L_{2}-1\end{array}$ \\
\hline & $\begin{array}{c}\frac{e^{-\lambda_{i 1} C(s)}\left(\lambda_{i 1} C(s)\right)^{n_{i 1}^{\prime}}}{n_{i 1}^{\prime} !}\left[1-\sum_{n_{i 3}^{\prime}=n_{i 3}}^{L_{2}-1} \frac{e^{-\lambda_{i 2} C(s)}\left(\lambda_{i 2} C(s)\right)^{\left(n_{i 3}^{\prime}-n_{i 3}\right)}}{\left(n_{i 3}^{\prime}-n_{i 3}\right) !}\right] \\
n_{i 1}=0,1 \leq n_{i 1}^{\prime} \leq L_{1}-1, n_{i 3}^{\prime}=L_{2}\end{array}$ \\
\hline & $\begin{array}{l}{\left[1-\sum_{n_{i 1}^{\prime}=0}^{L_{1}-1} \frac{e^{-\lambda_{i 1} C(s)}\left(\lambda_{i 1} C(s)\right)^{n_{i 1}^{\prime}}}{n_{i 1}^{\prime} !}\right] \cdot \frac{e^{-\lambda_{i 2} C(s)}\left(\lambda_{i 2} C(s)\right)^{\left(n_{i 3}^{\prime}-n_{i 3}\right)}}{\left(n_{i 3}^{\prime}-n_{i 3}\right) !}} \\
n_{i 1}=0, n_{i 1}^{\prime}=L_{1}, n_{i 3} \leq n_{i 3}^{\prime} \leq L_{2}-1\end{array}$ \\
\hline & $\begin{array}{l}{\left[1-\sum_{n_{i 1}^{\prime}=0}^{L_{1}-1} \frac{e^{-\lambda_{i 1} C(s)}\left(\lambda_{i 1} C(s)\right)^{n_{i 1}^{\prime}}}{n_{i 1}^{\prime} !}\right] \cdot\left[1-\sum_{n_{i 3}^{\prime}=n_{i 3}}^{L_{2}-1}\right.} \\
\left.\frac{e^{-\lambda_{i 2} C(s)}\left(\lambda_{i 2} C(s)\right)^{\left(n_{i 3}^{\prime}-n_{i 3}\right)}}{\left(n_{i 3}^{\prime}-n_{i 3}\right) !}\right], \quad n_{i 1}=0, n_{i 1}^{\prime}=L_{1}, n_{i 3}^{\prime}=L_{2}\end{array}$ \\
\hline & $\begin{array}{l}\frac{e^{-\lambda_{i 1} C(s)}\left(\lambda_{i 1} C(s)\right)^{n_{11}^{\prime}-n_{i 1}+1}}{\left(n_{i 1}^{\prime}-n_{i 1}+1\right) !} \cdot \frac{e^{-\lambda_{i 2} C(s)}\left(\lambda_{i 2} C(s)\right)^{n_{i 3}^{\prime}-n_{i 3}}}{\left(n_{i 3}^{\prime}-n_{i 3}\right) !} \\
n_{i 1} \geq 1, \max \left(n_{i 1}-1,1\right) \leq n_{i 1}^{\prime} \leq L_{1}-1, n_{i 3} \leq n_{i 3}^{\prime} \leq L_{2}-1\end{array}$ \\
\hline & $\begin{array}{l}\frac{e^{-\lambda_{i 1} C(s)}\left(\lambda_{i 1} C(s)\right)^{\left(n_{i 1}^{\prime}-n_{i 1}+1\right)}}{\left(n_{i 1}^{\prime}-n_{i 1}+1\right) !} \cdot\left[1-\sum_{n_{i 3}^{\prime}=n_{i 3}}^{L_{2}-1} \frac{e^{-\lambda_{i 2} C(s)}\left(\lambda_{i 2} C(s)\right)^{\left(n_{i 3}^{\prime}-n_{i 3}\right)}}{\left(n_{i 3}^{\prime}-n_{i 3}\right) !}\right] \\
n_{i 1} \geq 1, \max \left(n_{i 1}-1,1\right) \leq n_{i 1}^{\prime} \leq L_{1}-1, n_{i 3}^{\prime}=L_{2}\end{array}$ \\
\hline & $\begin{array}{l}{\left[1-\sum_{n_{i 1}^{\prime}=n_{i 1}-1}^{L_{11}-1} \frac{\left.e^{-\lambda_{i 1} C(s)}\left(\lambda_{i 1} C(s)\right)^{\left(n_{i 1}^{\prime}-n_{i 1}+1\right.}\right)}{\left(n_{i 1}^{\prime}-n_{i 1}+1\right) !}\right] \cdot \frac{e^{-\lambda_{i 2} C(s)}\left(\lambda_{i 2} C(s)\right)^{\left(n_{i 3}^{\prime}-n_{i 3}\right)}}{\left(n_{i 3}^{\prime}-n_{i 3}\right) !}} \\
\quad n_{i 1} \geq 1, n_{i 1}^{\prime}=L_{1}, n_{i 3} \leq n_{i 3}^{\prime} \leq L_{2}-1\end{array}$ \\
\hline & $\begin{array}{c}{\left[1-\sum_{n_{i 3}^{\prime}=n_{i 1}-1}^{L_{1}-1} \frac{e^{-\lambda_{i 1} C(s)}\left(\lambda_{i 1} C(s)\right)^{\left(n_{i 1}^{\prime}-n_{i 1}+1\right)}}{\left(n_{i 1}^{\prime}-n_{i 1}+1\right) !}\right] \cdot\left[1-\sum_{n_{i 3}^{\prime}=n_{i 3}}^{L_{2}-1}\right.} \\
\left.\frac{e^{\left.-\lambda_{i 2} C^{\prime} s\right)}\left(\lambda_{i 2} C(s)\right)^{\left(n_{i 3}^{\prime}-n_{i 3}\right)}}{\left(n_{i 3}^{\prime}-n_{i 3}\right) !}\right], \quad n_{i 1} \geq 1, n_{i 1}^{\prime}=L_{1}, n_{i 3}^{\prime}=L_{2}\end{array}$ \\
\hline
\end{tabular}

On the other hand, the key parameter used in the expression of state transition probabilities, $p_{i 2}=\mathrm{P}$ (no outstanding high-priority packets in the system), can be derived similarly as in Section 3.2.5. That is, we define $\overrightarrow{n_{1}}=\left(n_{11}, \ldots, n_{N 1}\right)$ as the state, where 
Table 1. State transition probabilities for the token scheduling protocol (continued).

\begin{tabular}{|c|c|}
\hline $\begin{aligned} & \left(n_{i 1} ; n_{i 2}, n_{i 3}\right) \\
\rightarrow & \left(n_{i 1}^{\prime} ; n_{i 2}^{\prime}, n_{i 3}^{\prime}\right)\end{aligned}$ & Transition probability for station $i$ \\
\hline \multirow[t]{2}{*}{$\begin{aligned} & \left(0 ; 1, n_{i 3}\right) \\
\rightarrow & \left(0 ; 1, n_{i 3}^{\prime}\right)\end{aligned}$} & $\begin{array}{l}e^{-\lambda_{i 1} C(s)} \cdot \frac{e^{-\lambda_{i 2} C(s)}\left(\lambda_{i 2} C(s)\right)^{\left(n_{i 3}^{\prime}-n_{i 3}+1\right)}}{\left(n_{i 3}^{\prime}-n_{i 3}+1\right) !} p_{i 2} \\
\quad \max \left(n_{i 3}-1,1\right) \leq n_{i 3}^{\prime} \leq L_{2}-1\end{array}$ \\
\hline & $\begin{array}{l}e^{-\lambda_{i 1} C(s)} \cdot\left[1-\sum_{n_{i 3}^{\prime}=n_{i 3}-1}^{L_{2}-1} \frac{e^{-\lambda_{i 2} C(s)}\left(\lambda_{i 2} C(s)\right)^{\left(n_{i 3}^{\prime}-n_{i 3}+1\right)}}{\left(n_{i 3}^{\prime}-n_{i 3}+1\right) !}\right] p_{i 2}, \\
\quad n_{i 3}^{\prime}=L_{2}\end{array}$ \\
\hline \multirow[t]{7}{*}{$\begin{aligned} & \left(0 ; 1, n_{i 3}\right) \\
\rightarrow & \left(n_{i 1}^{\prime} ; 0, n_{i 3}^{\prime}\right)\end{aligned}$} & $\begin{array}{l}e^{-\lambda_{i 1} C(s)} \cdot e^{-\lambda_{i 2} C(s)} \\
n_{i 1}^{\prime}=0, n_{i 3}=1, n_{i 3}^{\prime}=0\end{array}$ \\
\hline & $\begin{array}{l}e^{-\lambda_{i 1} C(s)} \cdot \frac{e^{-\lambda_{i 2} C(s)}\left(\lambda_{i 2} C(s)\right)^{\left(n_{i 3}^{\prime}-n_{i 3}+1\right)}}{\left(n_{i 3}^{\prime}-n_{i 3}+1\right) !}\left(1-p_{i 2}\right) \\
n_{i 1}^{\prime}=0, \max \left(n_{i 3}-1,1\right) \leq n_{i 3}^{\prime} \leq L_{2}-1\end{array}$ \\
\hline & $\begin{array}{l}e^{-\lambda_{i 1} C(s)} \cdot\left[1-\sum_{n_{i 3}^{\prime}=n_{i 3}-1}^{L_{2}-1} \frac{e^{-\lambda_{i 2} C(s)}\left(\lambda_{i 2} C(s)\right)^{\left(n_{i 3}^{\prime}-n_{i 3}+1\right)}}{\left(n_{i 3}^{\prime}-n_{i 3}+1\right) !}\right]\left(1-p_{i 2}\right), \\
\quad n_{i 1}^{\prime}=0, n_{i 3}^{\prime}=L_{2}\end{array}$ \\
\hline & $\begin{array}{l}\frac{e^{-\lambda_{i 1} C(s)}\left(\lambda_{i 1} C(s)\right)^{n_{i 1}^{\prime}}}{n_{i 1}^{\prime} !} \frac{e^{-\lambda_{i 2} C(s)}\left(\lambda_{i 2} C(s)\right)^{\left(n_{i 3}^{\prime}-n_{i 3}\right)}}{\left(n_{i 3}^{\prime}-n_{i 3}\right) !} \\
1 \leq n_{i 1}^{\prime} \leq L_{1}-1, n_{i 3} \leq n_{i 3}^{\prime} \leq L_{2}-1 \\
\end{array}$ \\
\hline & $\begin{array}{l}\frac{e^{-\lambda_{i 1} C(s)}\left(\lambda_{i 1} C(s)\right)^{n_{i 1}^{\prime}}}{n_{i 1}^{\prime} !} \cdot\left[1-\sum_{n_{i 1}^{\prime}=0}^{L_{1}-1} \frac{e^{-\lambda_{i 2} C(s)}\left(\lambda_{i 2} C(s)^{\left(n_{i 3}^{\prime} n_{i 3}\right)}\right.}{\left(n_{i 3}^{\prime}-n_{i 3}\right) !}\right] \\
1 \leq n_{i 1}^{\prime} \leq L_{1}-1, n_{i 3}^{\prime}=L_{2}\end{array}$ \\
\hline & $\begin{array}{l}{\left[1-\sum_{n_{i 1}^{\prime}=0}^{L_{1}-1} \frac{e^{-\lambda_{i 1} C(s)}\left(\lambda_{i 1} C(s)\right)^{n_{i 1}^{\prime}}}{n_{i 1}^{\prime} !}\right] \cdot \frac{e^{-\lambda_{i 2} C(s)}\left(\lambda_{i 2} C(s)\right)^{\left(n_{i 3}^{\prime}-n_{i 3}\right)}}{\left(n_{i 3}^{\prime}-n_{i 3}\right) !}} \\
n_{i 1}^{\prime}=L_{1}, n_{i 3} \leq n_{i 3}^{\prime} \leq L_{2}-1\end{array}$ \\
\hline & $\begin{array}{l}{\left[1-\sum_{n_{i 1}^{\prime}=0}^{L_{1}-1} \frac{e^{-\lambda_{i 1} C(s)}\left(\lambda_{i 1} C(s)\right)^{n_{i 1}^{\prime}}}{n_{i 1}^{\prime} !}\right] \cdot\left[1-\sum_{n_{i 1}^{\prime}=0}^{L_{11}-1}\right.} \\
\left.\frac{e^{-\lambda_{i 2} C(s)}\left(\lambda_{i 2} C(s)\right)^{\left(n_{i 3}^{\prime}-n_{i 3}\right)}}{\left(n_{i 3}^{\prime}-n_{i 3}\right) !}\right], \quad n_{i 1}^{\prime}=L_{1}, n_{i 3}^{\prime}=L_{2}\end{array}$ \\
\hline
\end{tabular}

$n_{j 1}$ is the number of high-priority packets at the end of each period, and the period is defined as the time interval between two successive packet transmissions. ${ }^{9}$ The state transition probabilities for station $i$ can then be approximated as:

$$
p_{n_{i 1} \rightarrow n_{i 1}^{\prime}}=\left\{\begin{array}{c}
E\left(\frac{e^{-\lambda_{i 1}\left(m+N W+K_{i d l e} W\right)}\left(\lambda_{i 1}\left(m+N W+K_{i d l e} W\right)\right)^{n_{i 1}^{\prime}}}{n_{i 1}^{\prime} !}\right) \\
n_{i 1}=0,0 \leq n_{i 1}^{\prime} \leq L_{1}-1 \\
1-\sum_{n_{i 1}^{\prime}=0}^{L_{1}-1} E\left(\frac{e^{-\lambda_{i 1}\left(m+N W+K_{i d l e} W\right)}\left(\lambda_{i 1}\left(m+N W+K_{i d l e} W\right)\right)^{n_{i 1}^{\prime}}}{n_{i 1}^{\prime} !}\right) \\
\frac{1}{\nu\left(\overrightarrow{\left.n_{1}\right)}\right.} E\left(e^{-\lambda_{i 1}\left(m+N W+K_{i d l e} W\right)}\right) \\
n_{i 1} \geq 1, n_{i 1}^{\prime}=n_{i 1}-1 \\
\left(1-\frac{1}{\nu\left(\overrightarrow{n_{1}}\right)}\right) \cdot E\left(\frac{e^{-\lambda_{i 1}\left(m+N W+K_{i d l e} W\right)}\left(\lambda_{i 1}\left(m+N W+K_{i d l e} W\right)\right)^{n_{i 1}^{\prime}-n_{i 1}}}{\left(n_{i 1}^{\prime}-n_{i 1}\right) !}\right) \\
+\frac{1}{\nu(\vec{n})} \cdot E\left(\frac{e^{-\lambda_{i 1}\left(m+N W+K_{i d l e} W\right)}\left(\lambda_{i 1}\left(m+N W+K_{i d l e} W\right)\right)^{n_{i 1}^{\prime}-n_{i 1}+1}}{\left(n_{i 1}^{\prime}-n_{i 1}+1\right) !}\right) \\
n_{i 1} \geq 1, n_{i 1} \leq n_{i 1}^{\prime} \leq L_{1}-1 \\
1-p_{n_{i 1} \rightarrow\left(n_{i 1}-1\right)}-\sum_{n_{i 1}^{\prime}=n_{i 1}}^{L_{1}-1} p_{n_{i 1} \rightarrow n_{i 1}^{\prime}} \\
n_{i 1} \geq 1, n_{i 1}^{\prime}=L_{1}
\end{array}\right.
$$


where $\nu\left(\overrightarrow{n_{1}}\right) \triangleq \sum_{i=1}^{N}\left(1-\delta\left(n_{i 1}\right)\right)$ is the number of stations with high-priority packets. Whether a high-priority packet at station $i$ will be transmitted next or not depends on the location of station $i$ in the downstream (relative to the token's current position). Due to the statistically identical behavior of all stations in a homogeneous system, the probability that a high-priority packet at a station gets transmitted in the next transmission interval is approximated as $\frac{1}{\nu(\vec{x})}$.

Let $\phi_{\overrightarrow{n_{1}}}$ be the equilibrium probability that the system is in state $\phi_{\overrightarrow{n_{1}}}$, then

$p_{i 2}=\phi_{\overrightarrow{0}}=P($ no outstanding high-priority packets at the end of a period $)$.

\subsubsection{Queue Length Distribution}

Given the cycle time distribution, the queue length distribution of high-priority packets, $\left\{q_{1}^{i}(j), 0 \leq j \leq L_{1}\right\}$, can be obtained by considering the queue as an $\mathrm{M} / \mathrm{G} / 1 / L_{1}$ queue with the service time density function $b_{i 1}(t)=T_{c}(t)$. (Again, this expression is approximate for a high-priority packet which arrives at an empty station.) The queue length distribution for low-priority packets, $\left\{q_{2}^{i}(j), 0 \leq j \leq L_{2}\right\}$, can be obtained with the same approach except that the service time cumulative distribution $B_{i 2}(t)$ is now approximated as:

$$
\begin{aligned}
B_{i 2}(t) & =q_{1}^{i}(0) \cdot\left[p_{i 2} D_{A_{i 1}}^{<2>}(t-m)+\sum_{k=1}^{\infty}\left(1-p_{i 2}\right)^{k} p_{i 2} D_{A_{i 2}}^{<2>} * C T_{c}^{(k)}(t-m)\right] \\
& +\sum_{j=1}^{L_{1}} q_{1}^{i}(j) \cdot\left[\sum_{k=0}^{\infty}\left(1-p_{i 2}\right)^{k} p_{i 2} D_{A_{i 2}}^{<2>} * C T_{c}^{(k+j)}(t-m)\right]
\end{aligned}
$$

for $t \geq m$, where $C T_{c}^{(k)}(t)$ is the cumulative distribution of the $k$-fold convolution of the density function of $T_{c} . \quad D_{A_{i 1}}^{<2>}$ and $D_{A_{i 2}}^{<2>}$ are derived similarly as in Sect.3.2.2. Statistically, a low-priority packet has to wait for the token's $(j+1)$-th visit ${ }^{10}$ with probability $q_{1}^{i}(j)$ (on the average) before competing with other stations for medium access. This low-priority packet will gain medium access with probability, $p_{i 2}$, that no high-priority packets exist in other stations.

\subsubsection{Probability of Packet Rejection and Distribution of Access Delay}

The probability of rejecting high-priority (low-priority) packets is $Q_{i}^{<1>}=q_{1}^{i}\left(L_{1}\right)$ $\left(Q_{i}^{<2>}=q_{2}^{i}\left(L_{2}\right)\right)$. The distribution of access delay experienced by high-priority packets can be expressed as:

$$
P\left(D_{A i}^{<1>} \leq t\right)=q_{1}^{i}(0) P\left(D_{A_{i 1}}^{<1>} \leq t\right)+\sum_{k=1}^{L_{1}-1} q_{1}^{i}(k) P\left(D_{A_{i 2}}^{<1>} \leq t\right) * P^{(k)}\left(T_{c} \leq t\right),
$$


and the distribution of access delay experienced by low-priority packets is approximated as

$$
\begin{aligned}
& P\left(D_{A i}^{<2>} \leq t\right)= \\
& q_{1}^{i}(0) \cdot\left\{q_{2}^{i}(0)\left[p_{i 2} P\left(D_{A_{i 1}}^{<2>} \leq t\right)+\sum_{k=1}^{\infty}\left(1-p_{i 2}\right)^{k} p_{i 2} P\left(D_{A_{i 2}}^{<2>} \leq t\right) * P^{(k)}\left(T_{c} \leq t\right)\right]\right. \\
&\left.\quad+\sum_{j=1}^{L_{2}-1} q_{2}^{i}(j) P\left(D_{A_{i 2}}^{<2>} \leq t\right) *\left[\sum_{k=0}^{\infty}\left(1-p_{i 2}\right)^{k} p_{i 2} P^{(k)}\left(T_{c} \leq t\right)\right]^{(j+1)}\right\} \\
&+\sum_{j=1}^{L_{1}} q_{1}^{i}(j) \cdot\left\{\sum_{k=0}^{L_{2}-1} q_{2}^{i}(k) P\left(D_{A_{i 2}}^{<2>} \leq t\right) * P^{(j)}\left(T_{c} \leq t\right)\right. \\
&\left.*\left[\sum_{\ell=0}^{\infty}\left(1-p_{i 2}\right)^{\ell} p_{i 2} P^{(\ell)}\left(T_{c} \leq t\right)\right]^{(k+1)}\right\} .
\end{aligned}
$$

\section{3. $\quad P_{i}$-Persistent Protocol}

\subsubsection{Definition and Transition of States}

The state of station $i, n_{i}\left(0 \leq n_{i} \leq L\right)$, is now defined as the number of outstanding packets at station $i$ at the end of each slot. The transition probability can then be expressed as

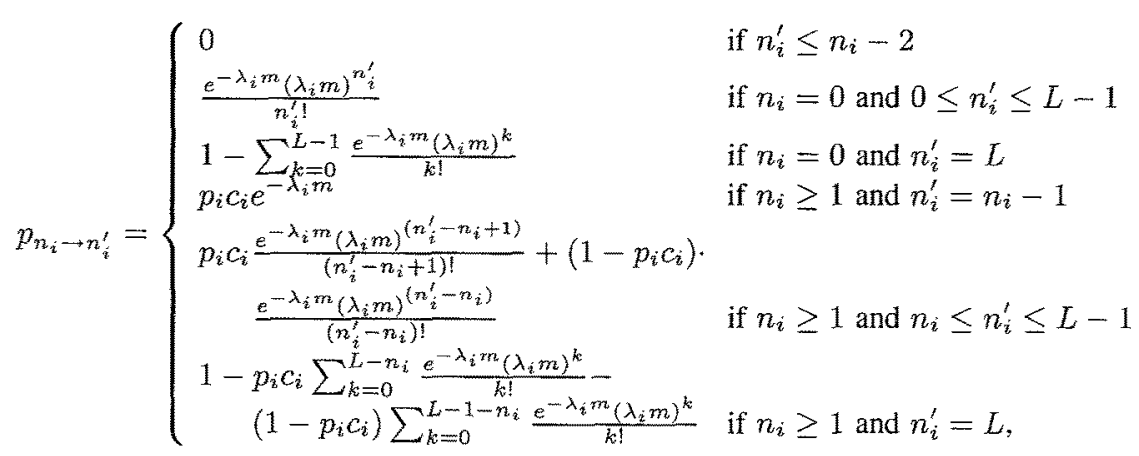

where $c_{i}$ is the probability that a slot seen by station $i$ is empty, and can be expressed similarly as in Eq.(18) except that $\pi_{i 1}$ should be replaced by $1-\pi_{i 0}$. Both $c_{i}$ and station $i$ 's equilibrium probability distribution can be obtained numerically. $D_{A_{i 1}}(t)$ for packets at the head of the queue is then given by

$$
D_{A_{i 1}}(t)=\sum_{k=0}^{\lfloor t / m\rfloor}\left(1-c_{i} p_{i}\right)^{k} c_{i} p_{i}
$$




\subsubsection{Queue Length Distribution, Probability of Packet Rejection, and Distribution of Access Delay}

To derive the queue length distribution, $\left\{q^{i}(j), 0 \leq j \leq L\right\}$, each station $i$ is modeled as an $\mathrm{M} / \mathrm{G} / 1 / L$ queue with the service time distribution $B_{i}(t)=D_{A_{i 1}}(t-m), t \geq m ; 0$, otherwise. (Note that the expression for $B_{i}(t)$ here is exact.) The probability of packet rejection is then given by $Q_{i}=q^{i}(L)$. The distribution of access delay can be expressed as

$$
P\left(D_{A} \leq t\right)=\sum_{k=0}^{L-1} q^{i}(k) P^{(k+1)}\left(D_{A_{i 1}} \leq t\right) .
$$

\subsection{Priority-Based $P_{i}$-persistent Protocol}

The state of station $i$ is now defined as $\left(n_{i 1}, n_{i 2}\right)\left(0 \leq n_{i 1} \leq L_{1}, 0 \leq n_{i 2} \leq L_{2}\right)$, where $n_{i 1}$ and $n_{i 2}$ are the number of high-priority and low-priority packets waiting at station $i$ at the end of each time slot, respectively. Expressions for the state transition probabilities are given in Table 2, where $c_{i}$ is the probability that a slot seen by station $i$ is empty, and is expressed as

$$
c_{i}=\left(1-\sum_{n_{i 2}=0}^{L_{2}} \sum_{n_{i 1}=1}^{L_{1}} \pi_{\left(n_{i 1}, n_{i 2}\right)} p_{i 1}-\sum_{n_{i 2}=1}^{L_{2}} \pi_{\left(0, n_{i 2}\right)} p_{i 2}\right)^{N-1} .
$$

Both $c_{i}$ and station $i$ 's equilibrium probability distribution can be obtained numerically.

The length distribution of the high-priority (low-priority) packet queue, $\left\{q_{1}^{i}(j), 0 \leq\right.$ $\left.j \leq L_{1}\right\},\left(\left\{q_{2}^{i}(j), 0 \leq j \leq L_{2}\right\}\right)$, can be obtained by considering the high-priority (lowpriority) queue as an $\mathrm{M} / \mathrm{G} / 1 / L_{1}$ (M/G/1/L $\left.L_{2}\right)$ queue with the service time distribution

$$
B_{i 1}(t)=D_{A_{i 1}}^{<1>}(t-m), \text { where } D_{A_{i 1}}^{<1>}(t)=\sum_{k=0}^{\lfloor t / m\rfloor}\left(1-c_{i} p_{i 1}\right)^{k} c_{i} p_{i 1},
$$

or

$$
B_{i 2}(t)=\sum_{k=0}^{L_{1}} q_{1}^{i}(k) D_{A_{i 1}}^{<1>(k)} * D_{A_{i 1}}^{<2>}(t-m)
$$

where

$$
D_{A_{i 1}}^{<2>}(t)=\sum_{k=0}^{\lfloor t / m\rfloor}\left(1-c_{i} p_{i 2}\right)^{k} c_{i} p_{i 2} .
$$

The probability of rejecting high-priority (low-priority) packets is then given by $Q_{i}^{<1>}=q_{1}^{i}\left(L_{1}\right)\left(Q_{i}^{<2>}=q_{2}^{i}\left(L_{2}\right)\right)$. The distribution of access delay experienced by a high-priority packet and a low-priority packet can be expressed as 
Table 2. State transition probabilities for the priority-based $P_{i}$-persistent protocol.

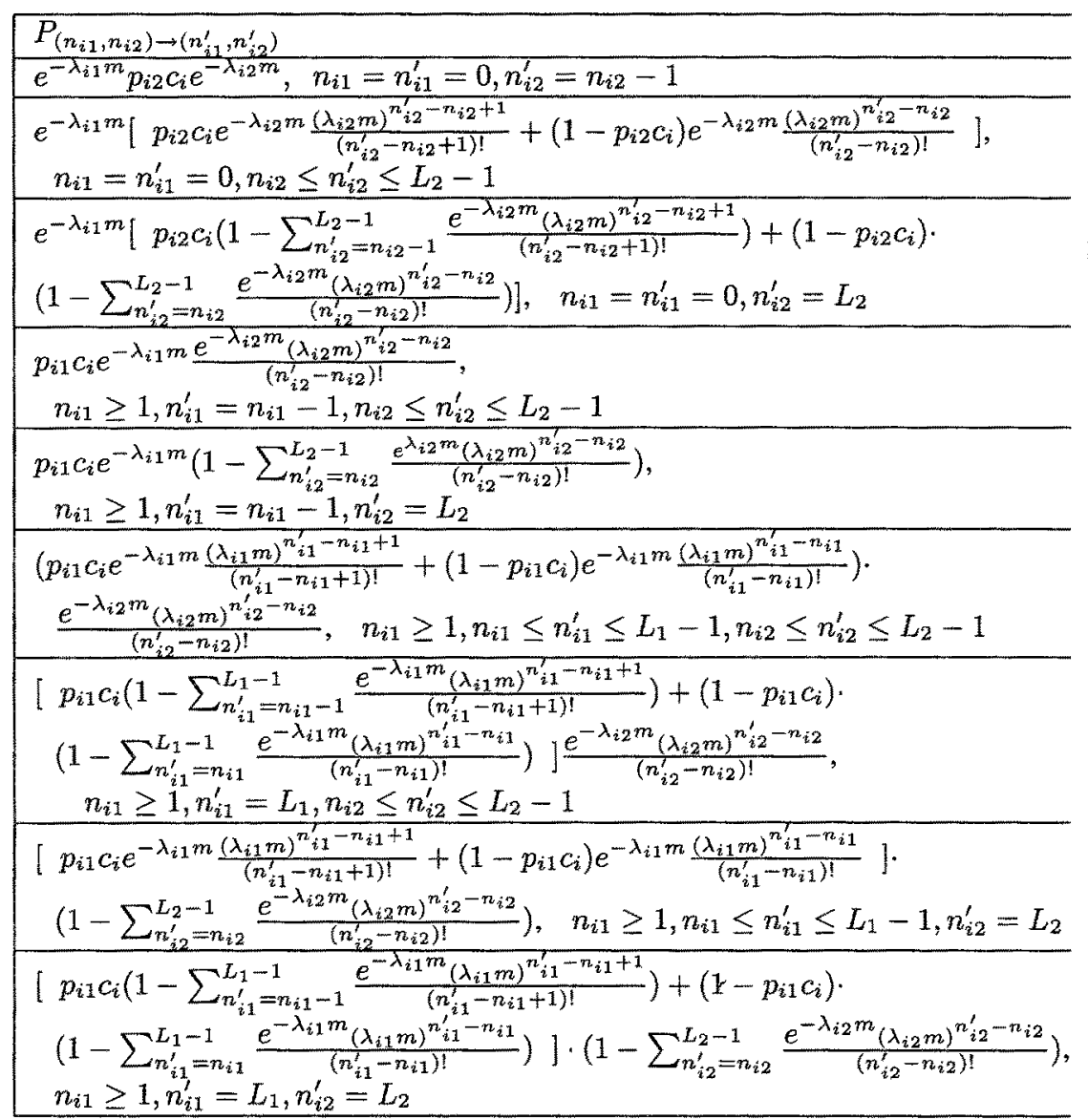

$$
P\left(D_{A_{i}}^{<1>} \leq t\right)=\sum_{k=0}^{L_{1}-1} q_{1}^{i}(k) P^{(k+1)}\left(D_{A 1_{i}} \leq t\right)
$$

and

$$
P\left(D_{A_{i}}^{<2>} \leq t\right)=\sum_{k=0}^{L_{1}} q_{1}^{i}(k)\left[P^{(k)}\left(D_{A_{i 1}}^{<1>} \leq t\right) * \sum_{\ell=0}^{L_{2}-1} q_{2}^{i}(\ell) P^{(\ell+1)}\left(D_{A_{i 1}}^{<2>} \leq t\right)\right],
$$

respectively.

\section{Numerical Examples}

We present some numerical results to illustrate the performance characteristics of the token-type and $P_{i}$-persistent-type protocols. In the single-buffer model, since the time 
each packet has to wait for other stations to transmit packets determines the quality of a contention protocol, we will analyze $D_{A_{i}}(t)$ (or, equivalently, $P_{d y n \mid d}$ ) and $T_{\epsilon}$. In case of multiple buffers at each station, we will analyze the performance trends resulting from the combined effects of contention protocols and local buffering strategies. Emphasis is placed on the effects of varying $L$ (or $L_{1}$ and $L_{2}$ ) on $P_{d y n}, T_{\epsilon}$, and $Q_{i}$.

Packet transmission time $(m)$, ring walk time $(N W)$, and the number of stations $(N)$ are chosen to be 1.0,0.04, and 10, respectively. All stations are assumed to have the same packet arrival rate $\lambda_{i}$. Two priority levels are assumed, because the performance characteristics are found to vary little even if the number of priority levels is greater than 2. Packet laxities are uniformly distributed in $[0,2 A L]$ where $A L$ is the average laxity. Packets with laxity $<2 r A L$ are classified as high-priority, and those with laxity $\geq 2 r A L$ are classified as low-priority, where $r=\frac{\lambda_{i 1}}{\lambda_{i}}$ is the fraction of high-priority packets.

Analytic results summarized below are obtained by solving the equation $\Pi P=\Pi$ with the Gaussian elimination method, computing cumulative probability distributions from their corresponding pdfs with Simpson's integration rule, and using FFT subroutines for time-frequency domain transformations. Event-driven simulations are also carried out for a 10-station homogeneous system, and are used to validate analytic results. For each configuration the simulation was run until we obtain results with a confidence level $95 \%$ for a maximum error of $5 \%$ of the value of measures of interest. The number of simulation runs needed to achieve the above confidence interval is based on the assumption that the parameter to be measured has a normal distribution with unknown mean and variance.

\subsection{Results from Single-Buffer Model}

We examine, for various traffic loads, the quality of contention protocols with $D_{A_{i}}(t)=$ $1-P_{d y n \mid t}$ and $T_{\epsilon}$ derived from the single-buffer model.

$D_{A_{i}}(t)$ and $D_{A_{i}}^{<1>}(t)$ are plotted in Figs. $2-3$ for the token passing and scheduling protocols, respectively. Table 3 and 4 give some numerical examples of $P_{d y n \mid t}^{<1>}, P_{d y n \mid t}^{<2>}$, and $T_{\epsilon}^{<1>}$ for both protocols. As was expected, $P_{d y n \mid t}$ and $T_{\epsilon}$ for the token passing protocol increase as the traffic increases. However, for all possible arrival rates, $D_{A_{i}}(t)=$ $1-P_{d y n \mid t}$ always approaches 1 in $t=N W+(N-1) m$ units of time. This is because the token will, in the worst case, re-visit a station within $N W+(N-1) m$ units of time after its last visit to the station. $T_{\epsilon}$ is thus bounded by the maximum cycle time, $N W+(N-1) m$. The simulation results agree very well with the analytic results for this protocol. (For clarity of presentation, the simulation results are only plotted for $\lambda_{i}=0.1$ in Fig. 2.)

The performance of the token scheduling protocol in delivering high-priority packets is significantly improved, as compared to the token passing protocol except when the traffic is extremely light. Note, however, that this improvement was made at the expense of performance in delivering low-priority packets (Table 3 ). The simulation results for the token scheduling protocol slightly differ from the analytic results (as compared to 


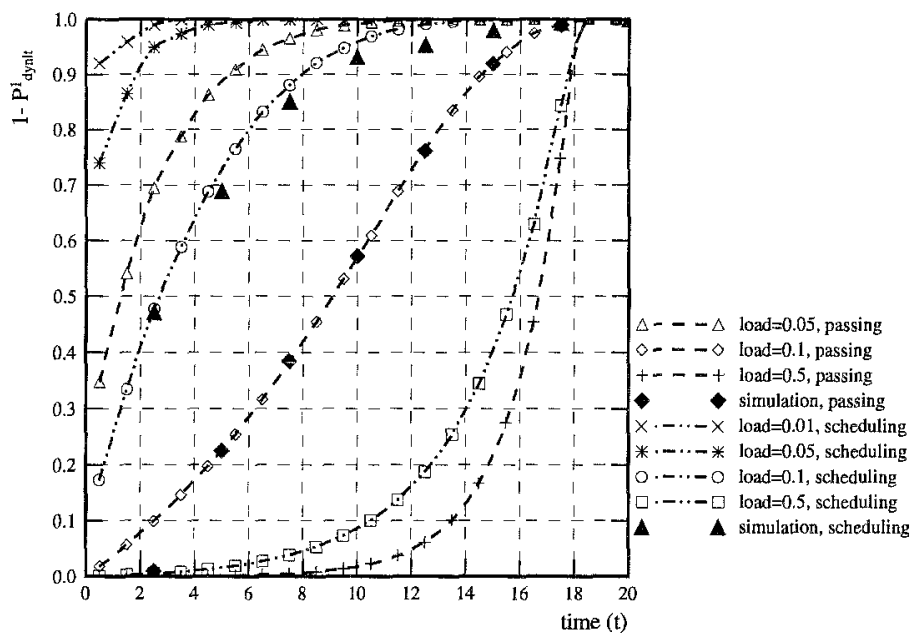

Figure 2. $1-P_{d y n \mid t}^{<1>}$ for token passing protocol and the token scheduling protocol. (Two packet priority levels exists with each contributing one half of the traffic.)

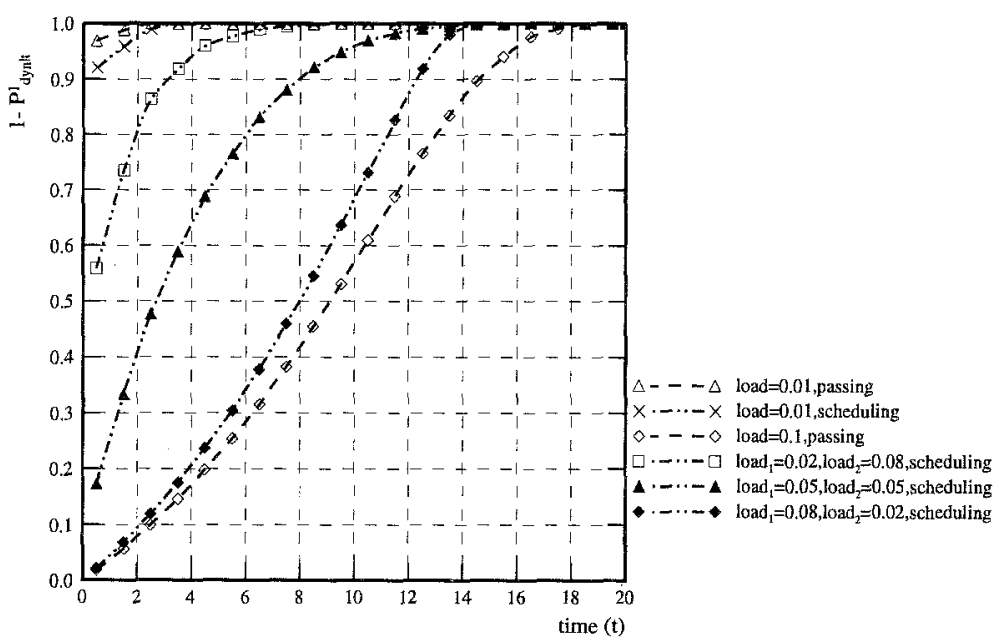

Figure $3 a$. Comparison of token passing protocol and token scheduling protocol with respect to $P_{d y n \mid t}$ under light and medium loads. 


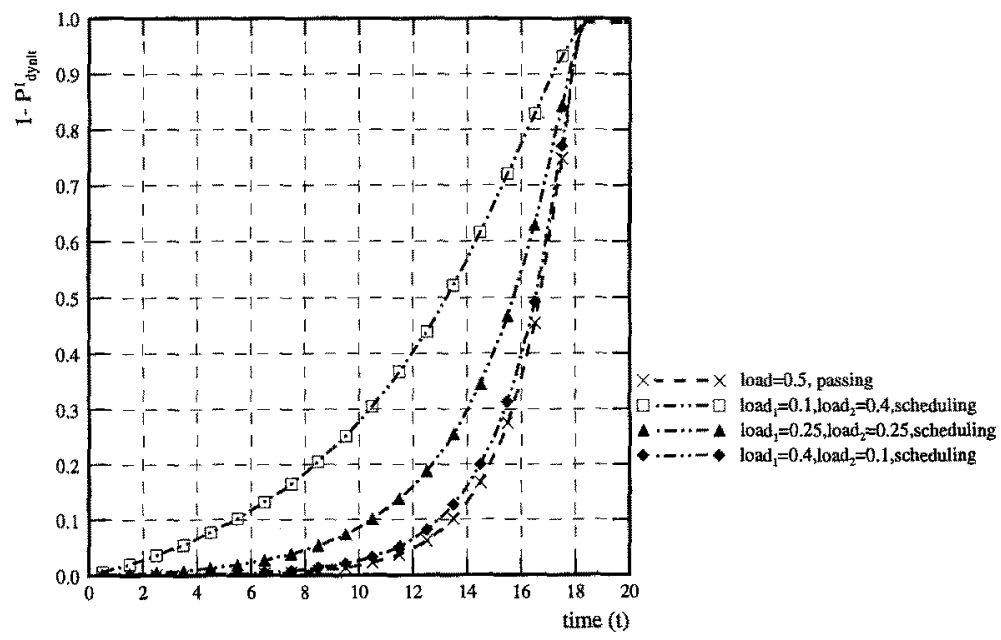

Figure $3 b$. Comparison of token passing protocol and token scheduling protocol with respect to $P_{d y n \mid t}$ under heavy loads.

Table 3. Comparison of token passing protocol and token scheduling protocol with respect to $P_{\text {dyn|t }}$.

\begin{tabular}{|c|c|c|c|c|}
\hline$\lambda_{i}$ & $t$ & Token passing & \multicolumn{2}{|c|}{ Token scheduling } \\
\cline { 3 - 5 } & & $P_{\text {dyn } \mid t}$ & $P_{\text {dynlt }}^{<1>}$ & $P_{\text {dyn }<2}^{<2>}$ \\
\hline 0.05 & 1 & 0.5571 & 0.1971 & 0.6258 \\
\cline { 2 - 5 } & 5 & 0.1143 & 0.0076 & 0.5213 \\
\cline { 2 - 5 } & 9 & 0.0151 & 0.0000 & 0.4431 \\
\cline { 2 - 5 } & 13 & 0.0005 & 0.0000 & 0.4119 \\
\cline { 2 - 5 } & 17 & 0.0000 & 0.0000 & 0.3850 \\
\cline { 2 - 5 } & 18.5 & 0.0000 & 0.0000 & 0.3642 \\
\hline 0.1 & 1 & 0.9632 & 0.7479 & 0.9721 \\
\cline { 2 - 5 } & 5 & 0.7749 & 0.2740 & 0.8237 \\
\cline { 2 - 5 } & 9 & 0.5085 & 0.0655 & 0.6436 \\
\cline { 2 - 5 } & 13 & 0.2008 & 0.0069 & 0.5274 \\
\cline { 2 - 5 } & 17 & 0.0176 & 0.0001 & 0.4898 \\
\cline { 2 - 5 } & 18.5 & 0.0000 & 0.0000 & 0.4277 \\
\hline
\end{tabular}

the discrepancy for the token passing protocol) due to approximations made in their derivation.

Fig. 3 also show the effects of varying the ratio of high-priority to low-priority packets on the performance of token scheduling protocol in delivering high-priority packets. For systems with very light traffic (e.g., $\lambda_{i}=0.01$ ), the performance is not significantly affected by this ratio, due to little contention faced by the high-priority packets in accessing 
Table 4. Comparison of token passing protocol and token scheduling protocol with respect to $T_{\varepsilon} . r$ is the ratio of high-priority to low-priority packets.

\begin{tabular}{|c|c|c|c|c|c|}
\hline$\lambda_{i}$ & $\epsilon$ & Token passing $\left(T_{\epsilon}\right)$ & \multicolumn{3}{|c|}{ Token scheduling $\left(T_{\epsilon}^{<1>}\right)$} \\
\cline { 3 - 6 } & & & $r=1: 4$ & $r=2: 2$ & $r=4: 1$ \\
\hline 0.05 & $10^{-3}$ & 12.37 & 3.89 & 6.20 & 6.27 \\
\cline { 2 - 6 } & $10^{-5}$ & 14.05 & 4.45 & 8.00 & 8.53 \\
\cline { 2 - 6 } & $10^{-7}$ & 14.18 & 4.79 & 8.23 & 8.76 \\
\cline { 2 - 6 } & $10^{-9}$ & 14.21 & 4.98 & 8.59 & 8.92 \\
\hline 0.1 & $10^{-3}$ & 18.05 & 9.42 & 15.25 & 15.29 \\
\cline { 2 - 6 } & $10^{-5}$ & 18.06 & 10.27 & 17.68 & 17.84 \\
\cline { 2 - 6 } & $10^{-7}$ & 18.08 & 10.42 & 18.01 & 18.04 \\
\cline { 2 - 6 } & $10^{-9}$ & 18.08 & 10.89 & 18.08 & 18.08 \\
\hline \multirow{3}{*}{0.5} & $10^{-3}$ & 18.06 & 17.98 & 18.04 & 18.04 \\
\cline { 2 - 6 } & $10^{-5}$ & 18.07 & 18.03 & 18.05 & 18.08 \\
\cline { 2 - 6 } & $10^{-7}$ & 18.08 & 18.05 & 18.06 & 18.14 \\
\cline { 2 - 6 } & $10^{-9}$ & 18.08 & 18.06 & 18.08 & 18.23 \\
\hline
\end{tabular}

the medium. (The numerical results are so close to one another for all traffic ratios that only one curve labeled as "load $=0.01$, scheduling" is plotted.) For systems with light to medium traffic (Fig. 3 (a), $\lambda_{i}=0.1$ ), the performance is substantially improved as the portion of high-priority packets decreases. Not only $1-P_{d y n \mid t}^{<1>}$ increases for a specific $t$ (Fig. 3 (a)), but also $T_{\epsilon}^{<1>}$ becomes smaller (Table 4). For systems with heavy traffic (Fig. 3 (b), $\lambda_{i}=0.5$ ), only $1-P_{d y n \mid t}^{<1>}$ increases as the portion of high-priority traffic decreases, except when the latter is very small. This indicates that there is an upper bound on the arrival rate of high-priority packets if every high-priority packet must be delivered within its deadline which is smaller than the maximum cycle time. On the other hand, even in the worst case where every station has an outstanding high-priority packet, $T_{\epsilon}^{<1>}$ is bounded by the maximum cycle time, $N W+(N-1)(m+N W)$.

Fig. 4 shows performance curves achievable with the best choice of $p_{i}$ for each possible $\lambda_{i}$. Except under extremely light traffic (in which case $c_{i} p_{i}$ is close to 1), the distribution of access delay approaches 1 at a very slow pace (Fig. 4). This accounts for the nonzero probability of a packet being delayed indefinitely. Consequently, while this protocol is suitable for handling bursty traffic, it is not appropriate for real-time applications. The simulation results are consistent with our analytic results for this protocol.

In Fig. 5, the performance experienced by high-priority packets under the prioritybased $P_{i}$-persistent protocol is compared to that under the $P_{i}$-persistent protocol for different ratios of high- and low- priority traffic. Each station persists to transmit highand low-priority packets with the best choice of $p_{i 1}$ and $p_{i 2}$ (calculated from the analytic model) for each specified $\lambda_{i}$. Under very light traffic, the difference is insignificant. (The numerical results are again so close to one another for all traffic ratios that only one curve labeled as "load $=0.01$ " is plotted.) For systems with medium to heavy traffic (Fig. 5, $\lambda=0.1$, the performance of the priority-based protocol is significantly better than that of the non priority-based one (labeled as "load $=0.1$ " in Fig. 5) if high-priority packets constitute only a small portion of the traffic. Under all circumstances, there is 


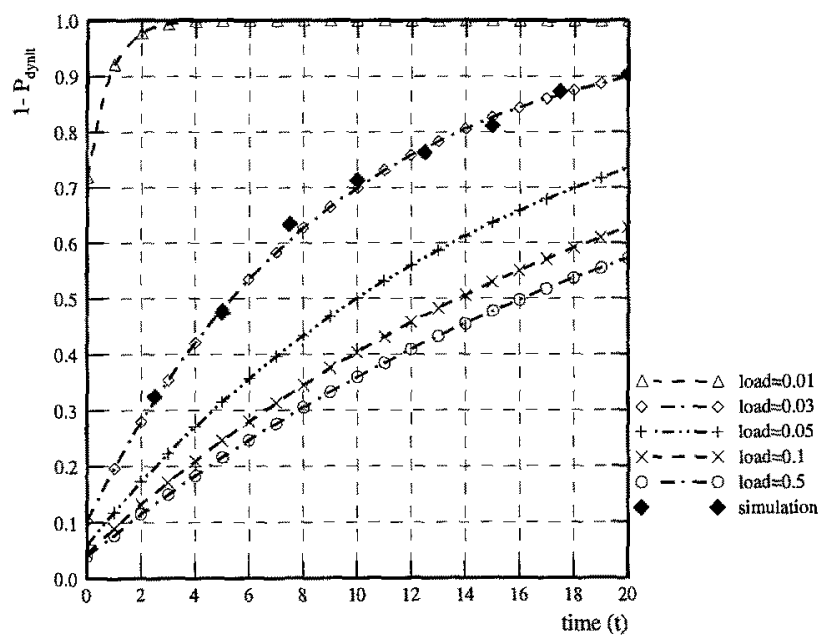

Figure 4. $1-P_{d y n}$ for $P_{i}$-persistent protocol with the best choice of $p_{i}$ for each $\lambda_{i}$

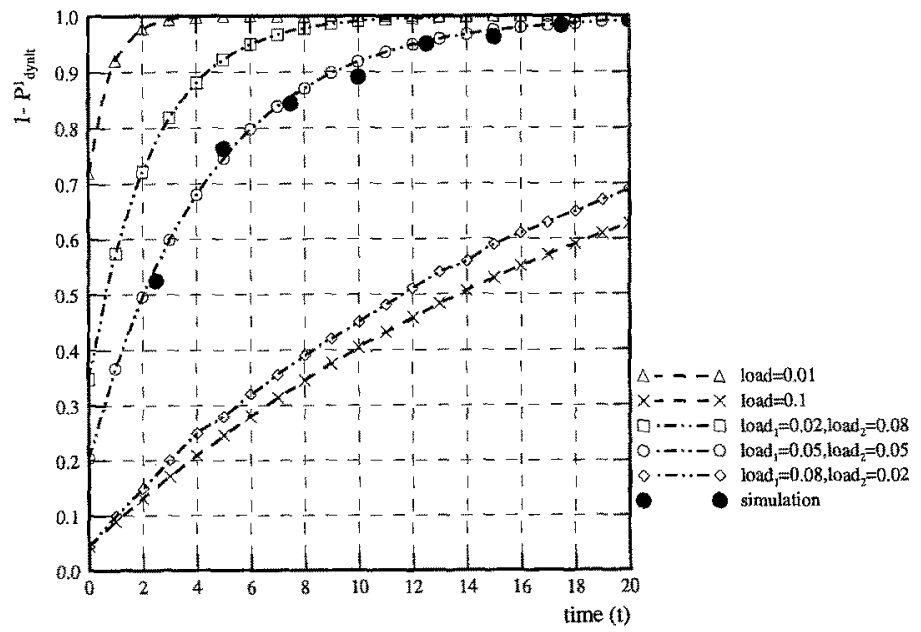

Figure 5. Comparison of $P_{i}$-persistent protocol and its priority-based variation. 
a nonzero probability, $\left(1-c_{i} p_{i}\right)^{k}$, that a packet will not be delivered within $k$ units of time. When the traffic is light to medium, and high-priority packets constitute only a small portion of the traffic, this probability is negligible and $T_{\epsilon}^{<1>}$ with $\varepsilon \leq 10^{-3}$ is acceptably small (Table 5). Consequently, the priority-based $P_{i}$-persistent protocol can be employed when high-priority packets constitute only a small portion of the traffic for soft real-time applications, where missing packet deadlines is undesirable but not disastrous. However, for hard real-time systems where missing deadlines is prohibited, some other mechanism which guarantees deterministic, bounded packet delivery times must be used.

Table 5. $T_{\varepsilon}$ of $P_{i}$-persistent protocol and its priority-based variation.

\begin{tabular}{|c|c|c|c|c|}
\hline$\lambda_{i}$ & $\epsilon$ & $P_{i}$-persistent & Priority $P_{i}$-persistent protocol $\left(T_{\epsilon}^{<1>}\right)$ \\
\cline { 3 - 5 } & & protocol $\left(T_{\epsilon}\right)$ & $r=1: 4$ & $r=2: 2$ \\
\hline \multirow{5}{*}{0.05} & $10^{-3}$ & 109.0 & 12.50 & 17.00 \\
\cline { 2 - 5 } & $10^{-5}$ & - & 19.60 & 29.00 \\
\cline { 2 - 5 } & $10^{-7}$ & - & 27.30 & 39.20 \\
\hline \multirow{3}{*}{0.1} & $10^{-3}$ & 144.2 & 15.50 & 26.70 \\
\cline { 2 - 5 } & $10^{-5}$ & - & 25.90 & 49.20 \\
\cline { 2 - 5 } & $10^{-7}$ & - & 36.10 & 67.00 \\
\hline \multirow{2}{*}{0.5} & $10^{-3}$ & 170.1 & 19.50 & 39.80 \\
\cline { 2 - 5 } & $10^{-5}$ & - & 33.00 & 66.70 \\
\cline { 2 - 5 } & $10^{-7}$ & - & 47.10 & 89.00 \\
\hline
\end{tabular}

\subsection{Results from Multiple-Buffer Model}

To study the performance differences between the single- and multiple- buffer models, and the combined effects of contention protocols and local buffering strategies on $P_{d y n}$, $T_{\epsilon}$ and $Q_{i}$, we derived numerical examples from multiple-buffer models with $A L=30$ and $r=0.2$. Both the analytic and simulation results indicate that the performance for the $P_{i}$-persistent-type protocols has trends similar to that for the token-type protocols (except all the measures $P_{d y n}, T_{\epsilon}$, and $Q_{i}$ have larger values for the same configuration) and thus is not included.

Fig. 6 plots $P_{d y n}^{<1>}$ and $P_{d y n}^{<2>}$ for the token passing protocol. Both measures increase as $L$ increases. This is because an arrived packet may be placed at the $j$-th position of the queue with probability $q^{i}(j-1)$ (Section 4.1.2) and has to wait for the transmission of $j-1$ packets ahead of it before competing with other stations for medium access. Besides, since time-constrained (high-priority) packets are less tolerant of the delay due to the FCFS-buffering strategy at each station, the increase in $P_{d y n}^{<1>}$ is more significant than that in $P_{d y n}^{<2>} . T_{\epsilon}$, as shown in the first column of Table 6 , increases with $L$ for the same reason. On the other hand, $Q_{i}$ decreases as $L$ increases, but the degree of improvement becomes insignificant as $L \geq 3$ (Fig. 7). The simulation results are again in good agreement with the analytic ones. (For clarity of presentation, simulation results are shown only in Fig. 6.) 


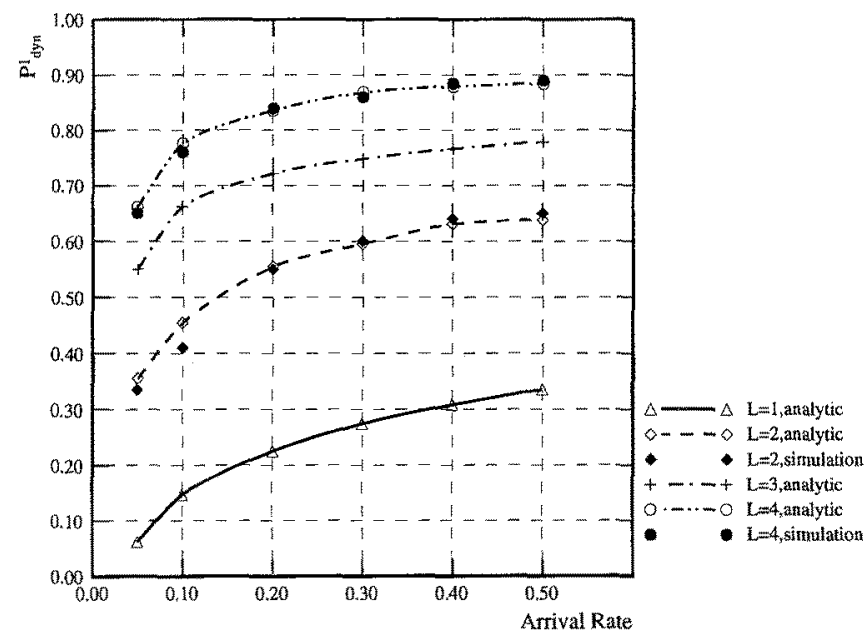

Figure 6a. $P_{d y n}^{<1>}$ for token passing protocol.

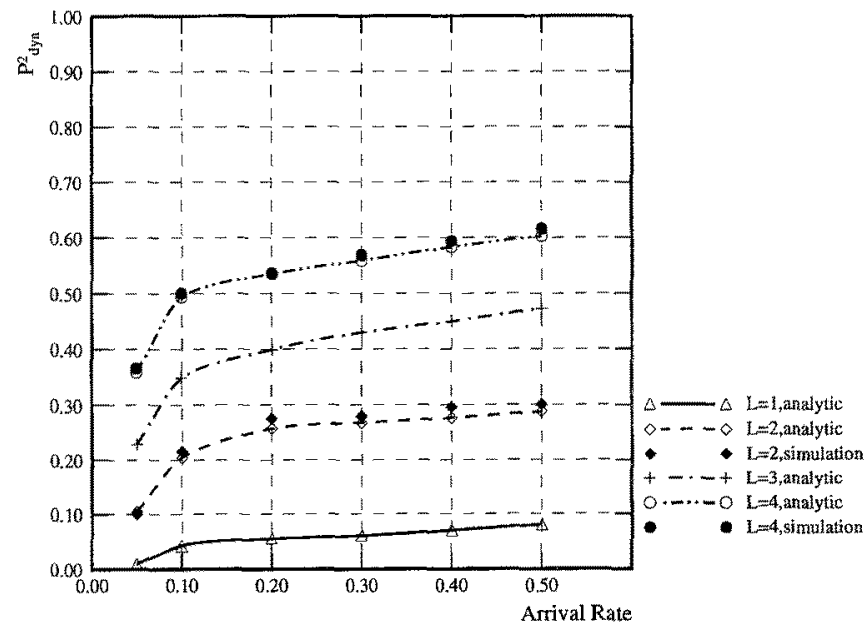

Figure $6 b, P_{d y n}^{<2>}$ for token passing protocol. 


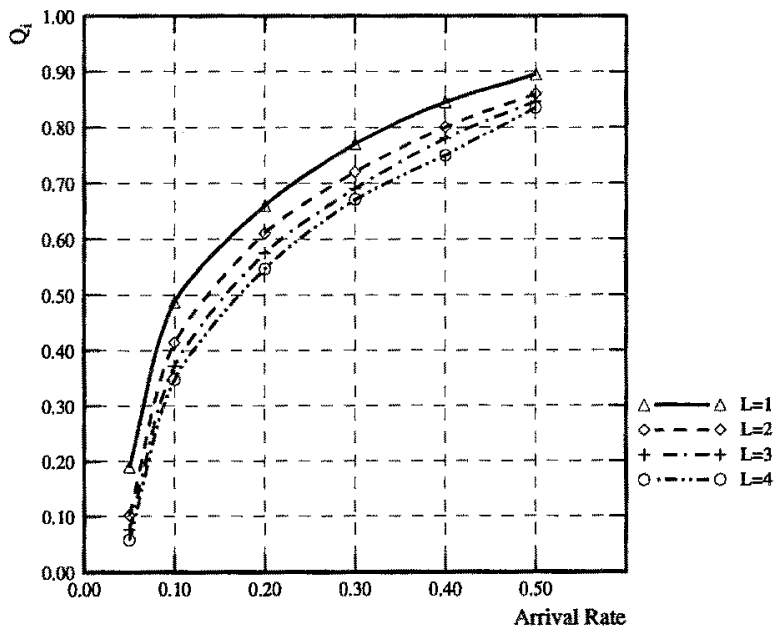

Figure 7. $Q_{i}$ for token passing protocol.

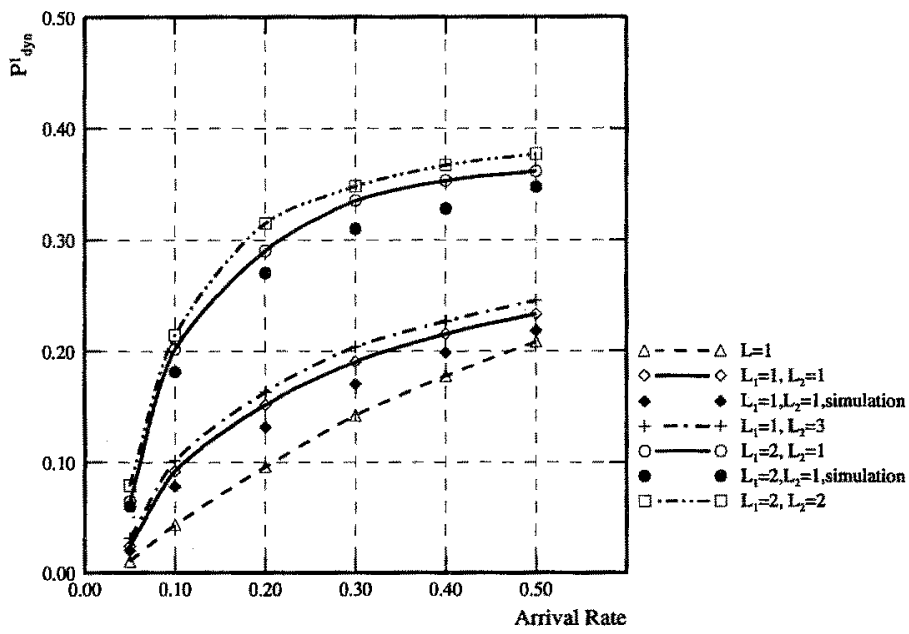

Figure 8a. $P_{d y n}^{<1>}$ for token scheduling protocol. 
Table 6. Effect of buffer size on $T_{\epsilon}$.

\begin{tabular}{|c|c|c|c|c|}
\hline$\lambda_{i}$ & Buffer size & Token passing & \multicolumn{2}{|c|}{ Token scheduling } \\
\cline { 2 - 5 } & & $T_{\epsilon} \epsilon=10^{-3}$ & $T_{\epsilon}^{<1>}$ & $T_{\epsilon}^{<>}$ \\
\hline 0.05 & $L=1$ & 12.37 & 3.89 & 21.76 \\
\cline { 2 - 5 } & $L=2\left(L_{1}=1, L_{2}=1\right)$ & 22.43 & 4.27 & 32.37 \\
\cline { 2 - 5 } & $L=3\left(L_{1}=1, L_{2}=2\right)$ & 34.26 & 4.32 & 40.28 \\
\cline { 2 - 5 } & $\bar{L}=3\left(L_{1}=2, L_{2}=1\right)$ & 34.26 & 10.98 & 46.32 \\
\cline { 2 - 5 } & $L=4\left(L_{1}=1, L_{2}=3\right)$ & 46.38 & 4.36 & 52.39 \\
\cline { 2 - 5 } 0.1 & $L=4\left(L_{1}=2, L_{2}=2\right)$ & 46.38 & 11.07 & 57.18 \\
\cline { 2 - 5 } & $L=2\left(L_{1}=1, L_{2}=1\right)$ & 18.05 & 9.42 & 29.43 \\
\cline { 2 - 5 } & $L=3\left(L_{1}=1, L_{2}=2\right)$ & 43.74 & 9.54 & 46.39 \\
\cline { 2 - 5 } & $L=3\left(L_{1}=2, L_{2}=1\right)$ & 48.32 & 9.60 & 58.23 \\
\cline { 2 - 5 } & $L=4\left(L_{1}=1, L_{2}=3\right)$ & 61.92 & 9.63 & 66.47 \\
\cline { 2 - 5 } & $L=4\left(L_{1}=2, L_{2}=2\right)$ & 61.92 & 17.82 & 82.45 \\
\hline 0.5 & $L=1$ & 18.06 & 17.98 & 41.89 \\
\cline { 2 - 5 } & $L=2\left(L_{1}=1, L_{2}=1\right)$ & 36.12 & 18.04 & 62.43 \\
\cline { 2 - 5 } & $\bar{L}=3\left(L_{1}=1, L_{2}=2\right)$ & 54.15 & 18.06 & 89.61 \\
\cline { 2 - 5 } & $L=3\left(L_{1}=2, L_{2}=1\right)$ & 54.15 & 27.76 & 99.75 \\
\cline { 2 - 5 } & $L=4\left(L_{1}=1, L_{2}=3\right)$ & 71.97 & 18.05 & 119.82 \\
\cline { 2 - 5 } & $L=4\left(L_{1}=2, L_{2}=2\right)$ & 71.97 & 27.97 & 136.38 \\
\hline
\end{tabular}

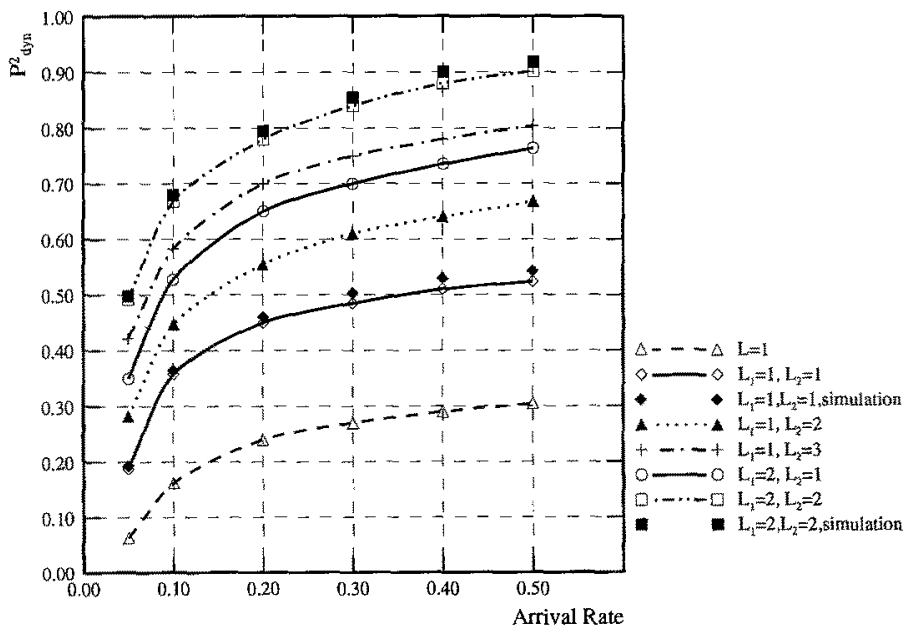

Figure $8 a . P_{d y n}^{<2>}$ for token scheduling protocol.

Figs. 8 (a) and 9 (a) plot $P_{d y n}^{<1>}$ and $Q_{i}^{<1>}$ of the token scheduling protocol for several combinations of $L_{1}$ and $L_{2}$, respectively. The second column of Table 6 gives numerical 
examples of $T_{\epsilon}^{<1>}$. Both $T_{\epsilon}^{<1>}$ and $P_{d y n}^{<1>}$ increase as $L_{1}$ increases, but the increase is not so pronounced as that for non priority-based protocols. This is because (1) a highpriority packet only has to wait for the transmission of high-priority packets (rather than all packets) arrived earlier before competing with other stations; (2) the access delay experienced by high-priority packets is much smaller than that in the non prioritybased case. $Q_{i}^{<1>}$ decreases as $L_{1}$ increases, and the degree of improvement is more pronounced as compared to that in the non priority-based case (Fig. 9 (a)). (The effect of varying $L_{2}$ on $Q_{i}^{<1>}$ is minimal, and only the curves for varying $L_{1}$ are shown in Fig. 9 (a).) This results from the fact that $E\left(B_{i}(t)\right)$ for the single-buffer case is substantially larger than $E\left(B_{i 1}(t)\right)$ in the two-buffer $\left(L_{1}=1, L_{2}=1\right.$ ) case (see Eq. (12)). ${ }^{11}$ The improvement on $Q_{i}^{<1>}$, however, levels off when $L_{1} \geq 3$.

An interesting finding is that $P_{d y n}^{<1>}, T_{\epsilon}^{<1>}$, and $Q_{i}^{<1>}$ remain almost the same (slightly degrade) as $L_{2}$ increases. This is because the size of the buffer holding low-priority packets has minimal effects on the performance of high-priority packets. A slight performance degradation results from the non-preemptive transmission policy in which a high-priority packet arriving at a station when a low-priority packet is being transmitted must wait for the in-progress transmission to complete. This possibility increases as $L_{2}$ increases. The analytic results for high-priority packets are always slightly worse than, but still in reasonably good agreement with, the corresponding simulation results.

Figs. 8 (b) and 9 (b) show the plots of $P_{d y n}^{<2>}$ and $Q_{i}^{<2>}$ for the token scheduling protocol. The third column of Table 6 gives numerical examples of $T_{\epsilon}^{<2>}$. Both $P_{d y n}^{<2>}$ and $T_{\epsilon}^{<2>}$ increase as $L_{1}$ or $L_{2}$ increases, and the degradation is more pronounced as $L_{1}$ increases. Another interesting consequence of this property is that $Q_{i}^{<2>}$ increases with $L_{1}$. This results from the fact that the access delay experienced by low-priority packets increases and more low-priority packets get queued in the system as more high-priority packets are accommodated.

The performance of contention protocols (in terms of $P_{d y n}$ and $T_{\varepsilon}$ ) derived from the multiple-buffer model exhibits similar trends as that derived from the single-buffer model except that the degree of performance improvement (or degradation) is furthered by local buffering strategies. Thus, if the quality of a contention protocol in terms of minimizing $P_{d y n}$ or $T_{\epsilon}$ is the main concern, a single-buffer model does provide a quantitative means of evaluating contention protocols. However, if the combined effects of contention protocols and local buffering strategies on the performance have to be considered, a multiple-buffer model must be used.

\section{Conclusion}

For two different types of contention protocols, we have developed both single- and multiple- buffer models to analytically evaluate two important parameters for real-time performance: the probability of dynamic failure, $P_{d y n}$, and the $\varepsilon$-bounded delivery time, $T_{\varepsilon}$. Another performance measure obtained as a byproduct is the probability of packet rejection, $Q_{i}$. Our analytic results are shown to agree well with the simulation results. 


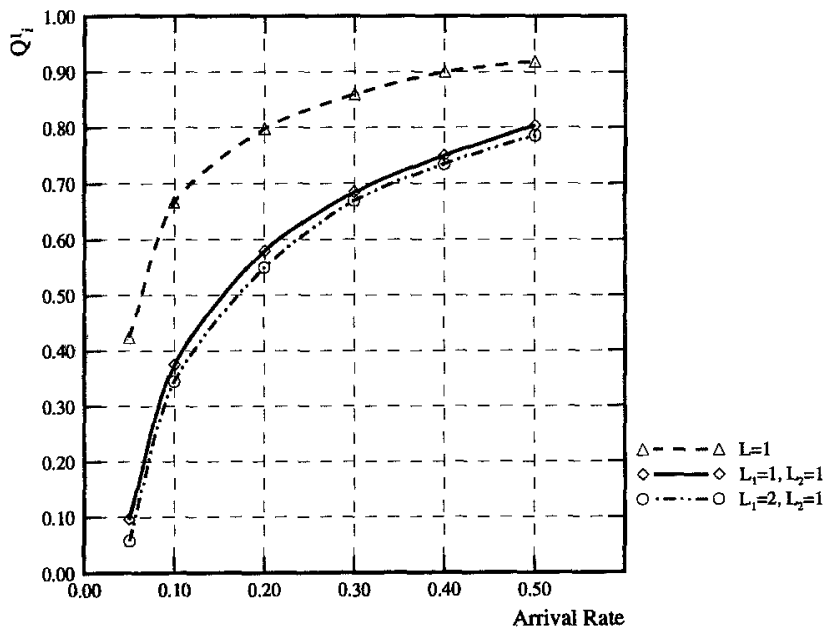

Figure $8 a . Q_{i}^{<1>}$ for token scheduling protocol.

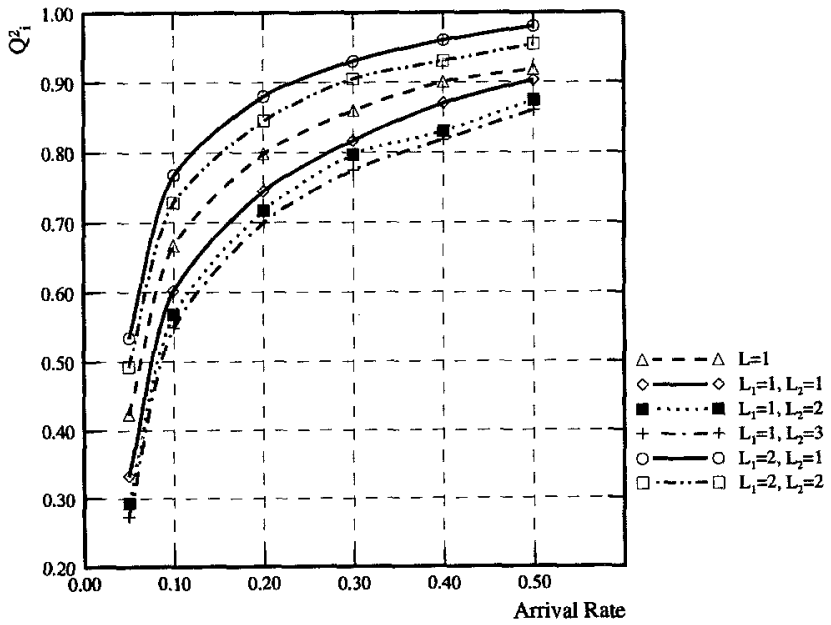

Figure $8 b . Q_{i}^{<2>}$ for token scheduling protocol. 
Our analyses have indicated the suitability of $P_{d y n}$ and $T_{\varepsilon}$ for assessing communication subsystems designed for real-time applications. For example, the token scheduling protocol is shown to outperform the token passing protocol with respect to both $P_{d y n}$ and $T_{\varepsilon}$ over a wide range of traffic. $P_{d y n}$ is minimized in the token scheduling protocol by giving tight-laxity packets priority over loose-laxity ones in accessing the medium, and the resulting improvement in meeting packet deadlines can be computed from the proposed analytic models. Our results also indicate the existence of a limit on the arrival rate of high-priority packets if every high-priority packet must be delivered by a specified time.

The $P_{i}$-persistent protocol is shown to be not attractive for real-time applications. The fact that there is a nonzero probability of a packet being indefinitely delayed is reflected in its unbounded $T_{\varepsilon}$ and relatively large $P_{d y n}$. On the other hand, both $P_{d y n}$ and $T_{\varepsilon}$ of its priority-based variation are shown to be acceptably small under certain conditions.

One interesting result is that $P_{d y n}$ and $T_{\varepsilon}$ obtained from the multiple-buffer model exhibit similar trends as those obtained from the single-buffer model except that the degree of performance improvement/degradation is furthered by local buffering strategies. Thus, the single-buffer model suffices for the evaluation of relative merits of different protocols based on $P_{d y n}$ and $T_{\varepsilon}$.

Analytically evaluating the performance of other real-time contention protocols, such as the virtual time CSMA/CD [29] and the window protocol implementing the minimumlaxity-first-transmission policy [30] would be an interesting extension of the work reported in this paper. Some form of state aggregation must be employed to reduce the size of the exponentially-growing state space for multiple-buffer models, which is currently under investigation.

\section{Notes}

1. This assumption is inherited from the assumption of homogeneous stations

2. One can easily extend this to the case with more than two priority levels.

3. There is no need to record the number of outstanding high-priority packets, since these packets must have the highest priority as the token comes to them, i.e., $p_{i 1}=1$.

4. $t_{2}-s_{2}$ is the number of outstanding low-priority packets in the system that had not been transmitted by the end of the last cycle time.

5. $k$ now represents the number of packets that have been transmitted.

6. For all stations to know the channel state (transmission, collision, or idle), the slot length must be at least $2 D_{p}+\gamma$, where $D_{p}$ is the end-to-end propagation delay, and $\gamma$ the time required for the detection of a collision by a station. The packet transmission time requires to be greater than $2 D_{p}+\gamma$, so each station can detect a collision.

7. The case in which there are more than two priority levels can be developed following the same approach.

8. Again, two cases are considered: (1) a packet arriving at a station without outstanding packets is placed at the head of the queue and gets transmitted upon the token's next visit; (2) a packet arriving at a station with outstanding packets is placed at the end of the queue, and will not be transmitted upon the token's next visit.

9. Note that the embedding point of the Markov chain is defined differently from that used in deriving the cycle time.

10. The count excludes those visits for priority polling 
11. As expressed in Eq. (15), the service time distribution $B_{i}(t)$ in the single-buffer case is a weighted sum of $D_{A_{i}}^{<1>}(t)$ and $D_{A_{i}}^{<2>}(t)$. Since $E\left(D_{A_{i}}^{<2>}(t)\right)$ is relatively large as a result of improving $D_{A_{i}}^{<1>}(t)$, $E\left(B_{i}(t)\right)$ becomes large.

\section{References}

1. S. Akhtar and A. K. Sood. Perfornance evaluation of CSMA/CD networks using markovian approach. GLOBECOM'88 IEEE Global Telecommunications Conference and Exhibition, pages 1108-1112, 1988.

2. Theodore K. Apostolopoulos and Emmanuel N. Protonotarios, Queneing analysis of buffered CSMA/CD protocols. IEEE Trans. on Communications, COM-34(9):898-905, September 1987.

3. M. J. Ferguson. Computation of the variance of the waiting time for token rings. IEEE J. Selected Areas Commun., SAC-4(6):775-782, September 1986.

4. M. J. Ferguson and Y. J. Aminetzah. Exact results for nonsymmetric token ring systems. IEEE Transactions on Communications, COM-33(3):223-231, March 1985.

5. Aura Ganz and Imrich Chlamtac. Queueing analysis of finite buffer token networks. Proc. ACM Sigmetrics, pages $30-36,1988$.

6. J. Gianini and D. R. Manfield. An analysis of symmetric polling systems with two priority classes. Performance Evaluations, 8:93-115, 1988.

7. Donald Gross and Carl Harris. Fundamentals of Queueing Theory. New York: Wiley, 1985.

8. Jeremiah F. Hayes. Modeling and Analysis of Computer Communication Networks. Plenum Press, 1984.

9. L. Kleinrock. Queueing Systems, Vol. 1: Theory. New York: Wiley, 1975.

10. H. Kobayashi. Modeling and analysis: an introduction to system performance evaluation mothodology. Addison-Wesley Pub. Co., 1978.

11. C. M. Krishna and K. G. Shin. Performance measures for multiprocessor controllers. Performance '83, pages 229-250, 1983. North-Holland, New York.

12. James F. Kurose, Mischa Schwartz, and Yechiam Yemini. Controlling window protocols for timeconstrained communication in multiple access network. IEEE Trans. on Comminications, 36(1):41-49, January 1988.

13. D. R. Manfield. Analysis of a priority polling system for two way traffic. IEEE Trans. Communications, COM-33(9):1001-1006, September 1985.

14. Biswanath Mukherjee and James $\mathrm{S}$. Meditch. The $p_{i}$-persistent protocol for unidirectional broadcast bus networks. IEEE Transactions on Communications, 36(12):1277-1286, December 1988.

15. J. W. M. Pang and R. W. Donaldson. Approximate delay analysis and results for asymmetric token passing and polling networks. IEEE J. Selected Areas Commun., SAC-4(6):783-793, September 1986.

16. I. Rubin and Z. Tsai, Performance analysis of a message priority based polling scheme. Proc. ICC, pages 22.1.1-22.1.5, June 1988 .

17. K. G. Shin, C. M. Krishna, and Y.-H. Lee. A unified method for evaluating real-time computer controllers and its application. IEEE Trans. on Automatic Control, AC-30(4):357-366, April 1985.

18. Kang G. Shin and C.-J. Hou. Analysis of three contention protocols in distributed real-time systems. IEEE Proc, of 11th Real-Time Systems Symposium, pages 136-145, December 1990.

19. J. K. Strosnider and T. E. Marchok. Responsive, deterministic IEEE 802.5 token ring scheduling. RealTime Systems Journal, 1(2):133-158, September 1989.

20. J. K. Strosnider, T. E. Marchok, and J. P. Lehoczky. Advanced real-time scheduling using the IEEE 802.5 token ring. Proceedings of IEEE Real-Time Systems Symposium, 1988.

21. Efstathios D. Sykas, Kionysios E. Karvelas, and Emmanuel N. Protonotarios. Queueing analysis of some buffered random multiple access schemes. IEEE Trans. on Communications, COM-34(8):790-798, August 1986.

22. H. Takagi. Analysis of Polling Systems. The MIT Press, Cambridge, Massachusetts, 1986.

23. H. Takagi. Queueing analysis of polling models. ACM Comput. Surveys, 20:5-28, March 1988.

24. Tetsuya Takine, Yutaka Takahashi, and Toshiharu Hasegawa. Exact analysis of asymmetric polling systems with single buffers. IEEE Transactions on Communications, 6(10):1119-1127, October 1988. Also in Performance '87, P.-J. Courtois, and G. Latouche, Eds. Elsevier North-Holland, Arnsterdam.

25. Andrew S. Tanenbaum. Computer Network. Prentice-Hall, Inc., 1989. 
26. F. A. Tobagi and V. B. Hunt. Performance analysis of carrier sense multiple access with collision detection. Computer Networks, 4:245-259, 1980.

27. Z. Tsai and I. Rubin. Performance of token schernes supporting delay-constrained priority traffic streams. IEEE Trans. Communications, COM-38(11):1994-2003, November 1990.

28. Cheng-Shong Wu and Victor O.K. Li. Delay analysis of buffered CSMA/CD protocols. GLOBECOM'88, IEEE Global Telecommunications Conference and Exhibition, pages 694-698, 1988.

29. Wei Zhao and K. Ramamitham. Virtual time CSMA protocols for hard real-time communications. IEEE Transactions on Software Engineering, SE-13(8):938-952, August 1987.

30. Wei Zhao, J. Stankovic, and K. Ramamitham. A window protocol for transmission of time constrained messages. Proceedings of the 8th International Conference on Distributed Computing Systems, pages 384-392, 1988. 